\title{
MATRIX POLYNOMIALS WITH COMPLETELY PRESCRIBED EIGENSTRUCTURE*
}

\author{
FERNANDO DE TERÁN ${ }^{\dagger}$, FROILÁN M. DOPICO $^{\dagger}$, AND PAUL VAN DOOREN D $^{\ddagger}$
}

\begin{abstract}
We present necessary and sufficient conditions for the existence of a matrix polynomial when its degree, its finite and infinite elementary divisors, and its left and right minimal indices are prescribed. These conditions hold for arbitrary infinite fields and are determined mainly by the "index sum theorem," which is a fundamental relationship between the rank, the degree, the sum of all partial multiplicities, and the sum of all minimal indices of any matrix polynomial. The proof developed for the existence of such polynomial is constructive and, therefore, solves a very general inverse problem for matrix polynomials with prescribed complete eigenstructure. This result allows us to fix the problem of the existence of $\ell$-ifications of a given matrix polynomial, as well as to determine all their possible sizes and eigenstructures.
\end{abstract}

Key words. matrix polynomials, index sum theorem, invariant polynomials, $\ell$-ifications, minimal indices, inverse polynomial eigenvalue problems

AMS subject classifications. 15A18, 15A21, 15A29, 15A54, 65F15, 65F18, 93B18

DOI. $10.1137 / 140964138$

1. Introduction. Matrix polynomials are essential in the study of dynamical problems described by systems of differential or difference equations with constant coefficient matrices

$$
P_{d} \Delta^{d} x(t)+\cdots+P_{1} \Delta x(t)+P_{0} x(t)=y(t)
$$

where $P_{i} \in \mathbb{F}^{m \times n}, \mathbb{F}$ is an arbitrary field, $P_{d} \neq 0$, and $\Delta^{j}$ denotes the $j$ th differential operator or the $j$ th difference operator, depending on the context. More precisely, the system (1.1) is associated to the matrix polynomial of degree $d$

$$
P(\lambda)=P_{d} \lambda^{d}+\cdots+P_{1} \lambda+P_{0} .
$$

The importance of matrix polynomials in different applications is widely recognized and is discussed in classic references [14, 18, 29], as well as in more recent surveys [32]. These references consider infinite fields, but it is worth emphasizing that matrix polynomials over finite fields are also of interest in applications like convolutional codes [12].

A matrix polynomial $P(\lambda)$ is regular when $P(\lambda)$ is square and the scalar polynomial $\operatorname{det} P(\lambda)$ has at least one nonzero coefficient. Otherwise $P(\lambda)$ is said to be singular. When $P(\lambda)$ is regular, the solutions of the system of differential/difference

*Received by the editors April 8, 2014; accepted for publication (in revised form) by F. Tisseur December 17, 2014; published electronically March 24, 2015. This research was partially supported by the Ministerio de Economía y Competitividad of Spain through grant MTM-2012-32542 and by the Belgian Network DYSCO (Dynamical Systems, Control, and Optimization), funded by the Interuniversity Attraction Poles Programme, initiated by the Belgian State, Science Policy Office.

http://www.siam.org/journals/simax/36-1/96413.html

†Department of Mathematics, Universidad Carlos III de Madrid, 28911 Leganés, Spain (fteran@math.uc3m.es, dopico@math.uc3m.es).

${ }^{\ddagger}$ Department of Mathematical Engineering, Université Catholique de Louvain, 1348 Louvainla-Neuve, Belgium (paul.vandooren@uclouvain.be). This work was developed while this author held a Chair of Excellence at Universidad Carlos III de Madrid in the academic year 2013-2014 supported by Banco de Santander. 
equations (1.1) depend on the eigenvalues and elementary divisors of $P(\lambda)$. When $P(\lambda)$ is singular, the solutions of (1.1) are also determined by the left and right null-spaces of $P(\lambda)$, which describe, respectively, constraints on the differential or difference equations to have compatible solutions, and also degrees of freedom in the solution set (see [37] for the case of polynomials of degree 1). The key concepts of left and right minimal indices are related to the null-spaces of $P(\lambda)[12,18]$ and so the elementary divisors, together with the left and right minimal indices, are called the complete eigenstructure of $P(\lambda)$ [35].

The problem of computing the complete eigenstructure of matrix polynomials has been widely studied since the 1970s (see [35] and the references therein) and, in particular, it has received a lot of attention in the last decade in order to find algorithms which are efficient, are stable, are able to preserve structures that are important in applications, may give reliable information on the complete eigenstructure under perturbations (stratifications), and are able to deal with large-scale matrix polynomial problems. This computational activity has motivated, in addition, the revision of many theoretical concepts on matrix polynomials to make them more amenable to computational purposes. To present a complete list of recent references on matrix polynomials is out of the scope of this work. So we just mention here the following small sample that may help the reader to look for many other recent references in this area: $[2,3,4,5,9,15,17,21,24,30,33]$.

In the context of the problems addressed in this paper, we would like to emphasize just a few aspects of the recent research on matrix polynomials. First, considerable activity has been devoted to the study of linearizations of matrix polynomials, due to the fact that the standard way of computing the eigenstructure of a matrix polynomial $P(\lambda)$ is through the use of linearizations $[14,35]$. Linearizations of a matrix polynomial $P(\lambda)$ are matrix polynomials of degree 1 having the same elementary divisors and the same dimension of the right and left null-spaces as $P(\lambda)$ [8, Lemma 2.3], [10, Theorem 4.1]. A second line of active recent research is the study of structured matrix polynomials arising in applications, which has revealed that linearizations cannot preserve the structure of some classes of structured polynomial eigenproblems (see [10, section 7] and the references therein). This drawback of linearizations has motivated the introduction of the new notion of $\ell$-ification in [10].

An $\ell$-ification of a matrix polynomial $P(\lambda)$ of degree $d$ is a matrix polynomial $Q(\lambda)$ of degree $\ell$ such that $P(\lambda)$ and $Q(\lambda)$ have the same elementary divisors and the same dimensions of the left and right null-spaces [10, Theorem 4.1]. An $\ell$-ification is strong if it additionally has the same elementary divisors at $\infty$ as $P(\lambda)$. Hence, (strong) linearizations are just (strong) $\ell$-ifications with $\ell=1$. Unlike what happens with strong linearizations, it has been shown that for a fixed value $\ell>1$ not every matrix polynomial of degree $d$ has a strong $\ell$-ification [10, Theorem 7.5]. This poses the problem of the existence of $\ell$-ifications, which is clearly related to the inverse problem of characterizing when a list of elementary divisors can be realized by a polynomial of given degree $\ell$ and given dimensions of its left and right null-spaces.

A third topic of current research we want to emphasize concerns "inverse polynomial eigenvalue problems of given degree," which have received attention in the literature since the 1970s [23, Theorem 5.2], have also been considered in classic references [14, Theorem 1.7], and recently have been studied in [17, Theorem 5.2], [19], and [30, section 5] (see also [22, section 9.1]), where new results that extend considerably the previous ones have been proved. Among all these references only [17] considers the existence and construction of matrix polynomials of given degree, with given 
elementary divisors, and given minimal indices, although only in the particular case of constructing polynomials with full rank, i.e., with only left or only right minimal indices but not with both. Quadratic inverse matrix polynomial eigenvalue problems have also been considered in [6] and, as a consequence of the study of quasi-canonical forms, in the ongoing works $[11,20]$.

In this paper, we consider the general inverse polynomial complete eigenstructure problem of given degree and we present necessary and sufficient conditions for the existence of a matrix polynomial of given degree, given finite and infinite elementary divisors, and given left and right minimal indices. These necessary and sufficient conditions are determined mainly by the "index sum theorem," a result discovered in the early 1990s for real polynomials [25, 28] and recently rediscovered, baptized, and extended to arbitrary fields in [10]. These necessary and sufficient conditions hold for arbitrary infinite fields and the proof of our main result is constructive, assuming that a procedure for constructing minimal bases is available (see [12, section 4]). Therefore, matrix polynomials with the desired properties can indeed be constructed, although the procedure on which our proof relies is not efficient from a computational point of view. Finally, the solution of this general inverse matrix polynomial eigenstructure problem allows us to completely solve the problem of the existence of $\ell$-ifications of a given polynomial $P(\lambda)$, as well as to determine all their possible sizes and eigenstructures.

The paper is organized as follows. In section 2, we review basic notions. Section 3 states and proves the main results of the paper, which requires us to develop some auxiliary lemmas. In section 4, existence, sizes, and minimal indices of $\ell$-ifications are studied. Finally, section 5 presents the conclusions and some directions of future research.

2. Preliminaries. The most important results in this paper hold for any infinite field $\mathbb{F}$. However, many auxiliary lemmas and definitions are valid for arbitrary fields $\mathbb{F}$ (finite or infinite). Therefore, we adopt the following convention for stating results: if the field is not explicitly mentioned in a certain result, then such result is valid for an arbitrary field. Otherwise, we will indicate explicitly that the field is infinite.

Next, we introduce some basic notation. The algebraic closure of the field $\mathbb{F}$ is denoted by $\overline{\mathbb{F}}$. By $\mathbb{F}[\lambda]$ we denote the ring of polynomials in the variable $\lambda$ with coefficients in $\mathbb{F}$, and $\mathbb{F}(\lambda)$ denotes the field of fractions of $\mathbb{F}[\lambda]$. Vectors with entries in $\mathbb{F}[\lambda]$ will be termed as vector polynomials and the degree of a vector polynomial is the highest degree of all its entries. $I_{n}$ denotes the $n \times n$ identity matrix and $0_{m \times n}$ the null $m \times n$ matrix.

Throughout the paper, we assume that the leading coefficient $P_{d}$ of a matrix polynomial $P(\lambda)=\sum_{i=0}^{d} P_{i} \lambda^{i}$ is nonzero and then we say that the degree of $P(\lambda)$ is $d$, denoted $\operatorname{deg}(P)=d$. Some references say in this situation that $P(\lambda)$ has "exact degree" $d[17]$, but we will not follow this convention for brevity.

Unimodular matrix polynomials often will be used. They are defined as follows [13].

DEFINITION 2.1. A square matrix polynomial $U(\lambda)$ is said to be unimodular if $\operatorname{det} U(\lambda)$ is a nonzero constant.

Note that the inverse of a unimodular matrix polynomial is also a unimodular matrix polynomial and that products of unimodular matrix polynomials are also unimodular. Therefore unimodular matrix polynomials form a transformation group and under this group a unique canonical form of an arbitrary matrix polynomial can be obtained [13]. This canonical form and some related definitions are introduced in Theorem 2.2 and Definition 2.3, respectively. 
Theorem 2.2. Let $P(\lambda)$ be an $m \times n$ matrix polynomial. Then there exist unimodular matrix polynomials $U(\lambda)$ and $V(\lambda)$ such that

$$
U(\lambda) P(\lambda) V(\lambda)=\left[\begin{array}{cccc|c}
p_{1}(\lambda) & 0 & \ldots & 0 & \\
0 & p_{2}(\lambda) & \ddots & \vdots & 0_{r \times(n-r)} \\
\vdots & \ddots & \ddots & 0 & \\
0 & \ldots & 0 & p_{r}(\lambda) & \\
\hline & 0_{(m-r) \times r} & & & 0_{(m-r) \times(n-r)}
\end{array}\right]=: D(\lambda)
$$

where $p_{1}(\lambda), \ldots, p_{r}(\lambda)$ are monic scalar polynomials and $p_{j}(\lambda)$ is a divisor of $p_{j+1}(\lambda)$, for $j=1, \ldots, r-1$. Moreover, the $m \times n$ diagonal matrix polynomial $D(\lambda)$ is unique.

DeFinition 2.3. The matrix $D(\lambda)$ in $(2.1)$ is called the Smith normal form of the matrix polynomial $P(\lambda)$. The polynomials $p_{j}(\lambda)$ are called the invariant polynomials of $P(\lambda)$. An invariant polynomial $p_{j}(\lambda)$ is trivial if $p_{j}(\lambda)=1$; otherwise $p_{j}(\lambda)$ is nontrivial (i.e., $\operatorname{deg}\left(p_{j}(\lambda)\right) \geq 1$ ).

$A$ finite eigenvalue of $P(\lambda)$ is a number $\alpha \in \overline{\mathbb{F}}$ such that $p_{j}(\alpha)=0$ for some $j=1, \ldots, r$. The partial multiplicity sequence of $P(\lambda)$ at the finite eigenvalue $\alpha$ is the sequence

$$
0 \leq \delta_{1}(\alpha) \leq \delta_{2}(\alpha) \leq \cdots \leq \delta_{r}(\alpha)
$$

such that $p_{j}(\lambda)=(\lambda-\alpha)^{\delta_{j}(\alpha)} q_{j}(\lambda)$ with $q_{j}(\alpha) \neq 0$, for $j=1, \ldots, r$. The elementary divisors of $P(\lambda)$ for the finite eigenvalue $\alpha$ are the collection of factors $(\lambda-\alpha)^{\delta_{j}(\alpha)}$ with $\delta_{j}(\alpha)>0$, including repetitions.

The number $r$ in (2.1) is the rank of $P(\lambda)$, which is denoted by $\operatorname{rank}(P)$.

The rank of $P(\lambda)$ is often called its "normal rank," but we will not use the adjective "normal" for brevity. An $m \times n$ matrix polynomial $P(\lambda)$ is said to have full rank if $\operatorname{rank}(P)=\min \{m, n\}$. Observe that some of the partial multiplicities at $\alpha$ appearing in (2.2) may be zero, but for defining the elementary divisors for $\alpha$ we consider only the partial multiplicities that are positive.

Given a matrix polynomial $P(\lambda)$ over a field $\mathbb{F}$ which is not algebraically closed, its elementary divisors for an eigenvalue $\alpha$ may not be polynomials over $\mathbb{F}$ according to Definition 2.3. To avoid this fact, we define, following [13, Chapter VI], the set of elementary divisors of $P(\lambda)$ (note that we do not specify here any eigenvalue) as the set of positive powers of monic irreducible scalar polynomials different from 1 over $\mathbb{F}$ appearing in the decomposition of each invariant polynomial $p_{j}(\lambda)$ of $P(\lambda)$, $j=1, \ldots, r$, into irreducible factors over $\mathbb{F}$. So, for instance, if $\mathbb{F}=\mathbb{R}$, then the elementary divisors of $P(\lambda)$ may be positive powers of real scalar polynomials of degree one or of real quadratic scalar polynomials with two complex conjugate roots.

Matrix polynomials may have infinity as an eigenvalue. Its definition is based on the so-called reversal matrix polynomial [21].

Definition 2.4. Let $P(\lambda)=\sum_{j=0}^{d} P_{j} \lambda^{j}$ be a matrix polynomial of degree $d$; the reversal matrix polynomial $\operatorname{rev} P(\mu)$ of $P(\lambda)$ is

$$
\operatorname{rev} P(\mu):=\mu^{d} P\left(\frac{1}{\mu}\right)=P_{d}+P_{d-1} \mu+\cdots+P_{0} \mu^{d} .
$$

Copyright $@$ by SIAM. Unauthorized reproduction of this article is prohibited. 
We emphasize that in this paper the reversal is always taken with respect to the degree of the original polynomial. Note that other options are considered in [10, Definition 2.12].

Definition 2.5. We say that $\infty$ is an eigenvalue of the matrix polynomial $P(\lambda)$ if 0 is an eigenvalue of $\operatorname{rev} P(\mu)$, and the partial multiplicity sequence of $P(\lambda)$ at $\infty$ is the same as that of the eigenvalue 0 in $\operatorname{rev} P(\mu)$. The elementary divisors $\mu^{\gamma_{j}}, \gamma_{j}>0$, for the eigenvalue $\mu=0$ of $\operatorname{rev} P(\mu)$ are the elementary divisors for $\infty$ of $P(\lambda)$.

Remark 2.6. It is well known that $\operatorname{rank}(P)=\operatorname{rank}(\operatorname{rev} P)$ (see, for instance, [22, Proposition 3.29]). Therefore, the length of the partial multiplicity sequence of $P(\lambda)$ at $\infty$ is equal to $\operatorname{rank}(P)$, as for any other eigenvalue of $P(\lambda)$. So, it follows from Definition 2.5 that $P(\lambda)$ is a matrix polynomial having no eigenvalues at $\infty$ if and only if its highest degree coefficient matrix $P_{d}$ has rank equal to $\operatorname{rank}(P)$.

The simple Lemma 2.7 will be used to establish certain conditions for the existence of matrix polynomials of given degree $d$.

Lemma 2.7. Let $P(\lambda)$ be a matrix polynomial with rank $r$. Then $P(\lambda)$ has strictly less than $r$ elementary divisors for the eigenvalue $\infty$.

Proof. Let $P(\lambda)=\sum_{j=0}^{d} P_{j} \lambda^{j}$ with $P_{d} \neq 0$. Then $\operatorname{rev} P(0)=P_{d} \neq 0$, and this implies that $\operatorname{rev} P(\lambda)$ has less than $r$ elementary divisors for the eigenvalue 0 , since otherwise $\operatorname{rev} P(0)=0$.

To avoid confusion with finite eigenvalues, the variable $\mu$ is used instead of $\lambda$ for the infinite eigenvalue and its elementary divisors, and $\gamma_{j}$ is used instead of $\delta_{j}$ for the partial multiplicities at $\infty$.

Remark 2.8. The partial multiplicity sequence at $\infty$ of a matrix polynomial $P(\lambda)$ as defined in Definition 2.5 should not be confused with the structural indices of $P(\lambda)$ at $\infty$ as defined, for instance, in [18, pp. 447, 450] (and in other references on linear system theory and control theory), although both concepts are related as we explain in this remark. The definition in [18, p. 447] is based on the Smith-MacMillan form [18, p. 443]. Given any $m \times n$ rational matrix $R(\lambda)$ there exist unimodular matrix polynomials $U(\lambda)$ and $V(\lambda)$ such that $U(\lambda) R(\lambda) V(\lambda)=$ $\operatorname{diag}\left(\theta_{1}(\lambda) / \psi_{1}(\lambda), \ldots, \theta_{r}(\lambda) / \psi_{r}(\lambda), 0, \ldots, 0\right)$, where each pair $\left(\theta_{i}(\lambda), \psi_{i}(\lambda)\right)$ is formed by coprime monic polynomials for $i=1, \ldots, r$, and $\theta_{j}(\lambda)$ divides $\theta_{j+1}(\lambda)$ and $\psi_{j+1}(\lambda)$ divides $\psi_{j}(\lambda)$, for $j=1, \ldots, r-1$. The matrix

$$
\operatorname{diag}\left(\theta_{1}(\lambda) / \psi_{1}(\lambda), \ldots, \theta_{r}(\lambda) / \psi_{r}(\lambda), 0, \ldots, 0\right)
$$

is called the Smith-MacMillan form of $R(\lambda)$ and is unique. The roots of $\theta_{1}(\lambda), \ldots, \theta_{r}(\lambda)$ are called the finite zeros of $R(\lambda)$, while the roots of $\psi_{1}(\lambda), \ldots, \psi_{r}(\lambda)$ are called the finite poles of $R(\lambda)$ [18, p. 446]. If $\alpha \in \overline{\mathbb{F}}$ is a finite zero or a finite pole (or simultaneously both) of $R(\lambda)$, then one can write $\theta_{i}(\lambda) / \psi_{i}(\lambda)=(\lambda-\alpha)^{\sigma_{i}(\alpha)}\left(\tilde{\theta}_{i}(\lambda) / \tilde{\psi}_{i}(\lambda)\right)$ with $\tilde{\theta}_{i}(\alpha) \neq 0, \tilde{\psi}_{i}(\alpha) \neq 0$, for $i=1, \ldots, r$, and the sequence $\sigma_{1}(\alpha) \leq \cdots \leq \sigma_{r}(\alpha)$ is the sequence of structural indices of $R(\lambda)$ at $\alpha$ [18, p. 447]. The sequence of structural indices of $R(\lambda)$ at $\infty$ is thus defined to be the sequence of structural indices of $R(1 / \lambda)$ at $0\left[18\right.$, p. 450]. Observe that if $\sigma_{1}(\alpha)<0$, then $\alpha$ is a pole, and that if $\sigma_{r}(\alpha)>0$, then $\alpha$ is a zero, where $\alpha$ may be $\infty$.

A matrix polynomial $P(\lambda)$ of degree $d>0$ is a special case of rational matrix and, therefore, we can define its zeros, poles, and structural indices as above. Since the Smith-MacMillan form of a matrix polynomial is just its Smith form (2.1), we see immediately that $P(\lambda)$ has no finite poles, that the finite zeros of $P(\lambda)$ are equal to the finite eigenvalues of $P(\lambda)$, and that the sequence of structural indices of $P(\lambda)$ at any finite zero $\alpha$ coincides with the partial multiplicity sequence of $P(\lambda)$ at the 
finite eigenvalue $\alpha$. The situation with infinity is not so simple. First, note that $P(1 / \lambda)$ has for sure a pole at zero and, so, $P(\lambda)$ has for sure a pole at $\infty$, but $P(\lambda)$ may have or not have an eigenvalue at $\infty$, as is clear from Remark 2.6. The key observation in this context is that the Smith form of the reversal polynomial rev $P(\lambda)=\lambda^{d} P(1 / \lambda)$ is just $\lambda^{d}$ times the Smith-MacMillan form of the rational matrix $P(1 / \lambda)$. This implies that if $\sigma_{1}(\infty) \leq \cdots \leq \sigma_{r}(\infty)$ is the sequence of structural indices of $P(\lambda)$ at $\infty$, then $0 \leq d+\sigma_{1}(\infty) \leq \cdots \leq d+\sigma_{r}(\infty)$ are the multiplicities of 0 as a root of the invariant polynomials of rev $P(\lambda)$. Therefore, $\infty$ is an eigenvalue of $P(\lambda)$ if and only if $0<d+\sigma_{i}(\infty)$ for at least one $i=1, \ldots, r$ and, in this case, $0 \leq d+\sigma_{1}(\infty) \leq \cdots \leq d+\sigma_{r}(\infty)$ is the partial multiplicity sequence of $P(\lambda)$ at the eigenvalue $\infty$. Observe that in fact $0=d+\sigma_{1}(\infty)$ as a consequence of Lemma 2.7 or simply because the largest order of the pole at $\infty$ of a matrix polynomial of degree $d$ is precisely its degree.

An $m \times n$ matrix polynomial $P(\lambda)$ whose rank $r$ is smaller than $m$ and/or $n$ has nontrivial left and/or right null-spaces over the field $\mathbb{F}(\lambda)$ :

$$
\begin{aligned}
& \mathcal{N}_{\ell}(P):=\left\{y(\lambda)^{T} \in \mathbb{F}(\lambda)^{1 \times m}: y(\lambda)^{T} P(\lambda) \equiv 0^{T}\right\}, \\
& \mathcal{N}_{r}(P):=\left\{x(\lambda) \in \mathbb{F}(\lambda)^{n \times 1}: P(\lambda) x(\lambda) \equiv 0\right\} .
\end{aligned}
$$

It is well known that every subspace $\mathcal{V}$ of $\mathbb{F}(\lambda)^{n}$ has bases consisting entirely of vector polynomials. In order to define the singular structure of $P(\lambda)$, we need to introduce minimal bases of $\mathcal{V}$.

Definition 2.9. Let $\mathcal{V}$ be a subspace of $\mathbb{F}(\lambda)^{n}$. A minimal basis of $\mathcal{V}$ is a basis of $\mathcal{V}$ consisting of vector polynomials whose sum of degrees is minimal among all bases of $\mathcal{V}$ consisting of vector polynomials.

It can be seen $[12,18]$ that the ordered list of degrees of the vector polynomials in any minimal basis of $\mathcal{V}$ is always the same. These degrees are then called the minimal indices of $\mathcal{V}$. This leads to the definition of the minimal indices of a matrix polynomial.

DeFINITION 2.10. Let $P(\lambda)$ be an $m \times n$ singular matrix polynomial with rank $r$ over a field $\mathbb{F}$, and let the sets $\left\{y_{1}(\lambda)^{T}, \ldots, y_{m-r}(\lambda)^{T}\right\}$ and $\left\{x_{1}(\lambda), \ldots, x_{n-r}(\lambda)\right\}$ be minimal bases of $\mathcal{N}_{\ell}(P)$ and $\mathcal{N}_{r}(P)$, respectively, ordered so that $0 \leq \operatorname{deg}\left(y_{1}\right) \leq \cdots \leq$ $\operatorname{deg}\left(y_{m-r}\right)$ and $0 \leq \operatorname{deg}\left(x_{1}\right) \leq \cdots \leq \operatorname{deg}\left(x_{n-r}\right)$. Let $\eta_{i}=\operatorname{deg}\left(y_{i}\right)$ for $i=1, \ldots, m-r$ and $\varepsilon_{j}=\operatorname{deg}\left(x_{j}\right)$ for $j=1, \ldots, n-r$. Then the scalars $\eta_{1} \leq \eta_{2} \leq \cdots \leq \eta_{m-r}$ and $\varepsilon_{1} \leq \varepsilon_{2} \leq \cdots \leq \varepsilon_{n-r}$ are, respectively, the left and right minimal indices of $P(\lambda)$.

In order to give a practical characterization of minimal bases, we introduce Definition 2.11. In the following, when referring to the column (resp., row) degrees $d_{1}, \ldots, d_{n}$ (resp., $d_{1}^{\prime}, \ldots, d_{m}^{\prime}$ ) of an $m \times n$ matrix polynomial $P(\lambda)$, we mean that $d_{j}$ (resp., $d_{j}^{\prime}$ ) is the degree of the $j$ th column (resp., row) of $P(\lambda)$.

Definition 2.11. Let $N(\lambda)$ be an $n \times r$ matrix polynomial with column degrees $d_{1}, \ldots, d_{r}$. The highest-column-degree coefficient matrix of $N(\lambda)$, denoted by $N_{h c}$, is the $n \times r$ constant matrix whose $j$ th column is the coefficient of $\lambda^{d_{j}}$ in the jth column of $N(\lambda) . N(\lambda)$ is said to be column reduced if $N_{h c}$ has full column rank.

Similarly, let $M(\lambda)$ be an $r \times n$ matrix polynomial with row degrees $d_{1}, \ldots, d_{r}$. The highest-row-degree coefficient matrix of $M(\lambda)$, denoted by $M_{h r}$, is the $r \times n$ constant matrix whose jth row is the coefficient of $\lambda^{d_{j}}$ in the jth row of $M(\lambda) . M(\lambda)$ is said to be row reduced if $M_{h r}$ has full row rank.

Remark 2.12. Note that the rank of an $n \times r$ column reduced matrix polynomial $N(\lambda)$ is $r$ [18, Chapter 6]. So, column reduced matrix polynomials have full column 
rank. A similar observation holds for row reduced matrix polynomials and full row rank.

The next example illustrates the difference between the leading coefficient of a matrix polynomial and its highest-column-degree and highest-row-degree coefficient matrices.

Example 2.13. Consider the following matrix polynomial with degree 3:

$$
P(\lambda)=\left[\begin{array}{cc}
0 & \lambda^{3}+\lambda^{2} \\
\lambda+1 & 3 \lambda \\
\lambda^{2} & 4
\end{array}\right]
$$

Then the leading coefficient, $P_{3}$, the highest-column-degree coefficient, $P_{h c}$, and the highest-row-degree coefficient, $P_{h r}$, of $P(\lambda)$ are

$$
P_{3}=\left[\begin{array}{ll}
0 & 1 \\
0 & 0 \\
0 & 0
\end{array}\right], \quad P_{h c}=\left[\begin{array}{ll}
0 & 1 \\
0 & 0 \\
1 & 0
\end{array}\right], \quad P_{h r}=\left[\begin{array}{ll}
0 & 1 \\
1 & 3 \\
1 & 0
\end{array}\right] .
$$

Theorem 2.14 provides a characterization of those matrix polynomials whose columns or rows are minimal bases of the subspaces they span. Theorem 2.14 is a minor variation of [12, Main Theorem (2), p. 495], which we think is easier to use in practice when $\mathbb{F}$ is not algebraically closed. In [18, Theorem 6.5-10], we can find the corresponding result stated only for the complex field, but the proof remains valid for any algebraically closed field.

THEOREM 2.14. The columns (resp., rows) of a matrix polynomial $N(\lambda)$ over a field $\mathbb{F}$ are a minimal basis of the subspace they span if and only if $N\left(\lambda_{0}\right)$ has full column (resp., row) rank for all $\lambda_{0} \in \overline{\mathbb{F}}$ and $N(\lambda)$ is column (resp., row) reduced.

Proof. We prove the result only for columns, since for rows it is similar. Let $n \times r$ be the size of $N(\lambda)$. According to [12, Main Theorem (2), p. 495], the columns of $N(\lambda)$ are a minimal basis if and only if $N(\lambda)$ has full column rank modulo $p(x)$ for all irreducible $p(x) \in \mathbb{F}[\lambda]$ and $N(\lambda)$ is column reduced. So, we only need to prove that $N(\lambda)$ has full column rank modulo $p(x)$ for all irreducible $p(x) \in \mathbb{F}[\lambda]$ if and only if $N\left(\lambda_{0}\right)$ has full column rank for all $\lambda_{0} \in \overline{\mathbb{F}}$. Note that " $N(\lambda)$ has full column rank modulo $p(x)$ for all irreducible $p(x) \in \mathbb{F}[\lambda]$ " is equivalent to "the greatest common divisor of all $r \times r$ minors of $N(\lambda)$ is 1" [12, p. 496], which is equivalent to "the Smith normal form of $N(\lambda)$ is $\left[\begin{array}{ll}I_{r} & 0\end{array}\right]^{T}$ over $\mathbb{F}^{\prime \prime}[13$, Chapter VI]. But the Smith forms of $N(\lambda)$ over $\mathbb{F}$ and $\overline{\mathbb{F}}$ are the same and the latter statement is obviously equivalent to "N( $\left.\lambda_{0}\right)$ has full column rank for all $\lambda_{0} \in \overline{\mathbb{F}}$."

Remark 2.15. In this paper, for brevity, we often say that a $p \times q$ matrix polynomial $N(\lambda)$ with $p \geq q$ (resp., $p<q$ ) is a minimal basis if the columns (resp., rows) of $N(\lambda)$ are a minimal basis of the subspace they span.

Lemma 2.16 gathers some known simple properties that will be used in what follows. These properties are by no means new. For instance, Lemma 2.16(b) can be found in [1, Lemma 3.5] and Lemma 2.16(c) can be found in [31], although a simpler proof can be obtained using the ideas in [26]. We include proofs of these results just to keep the paper self-contained.

Lemma 2.16. Let $N(\lambda)$ be a matrix polynomial over a field $\mathbb{F}$ :

(a) If the columns of $N(\lambda)$ form a minimal basis and $R$ is a nonsingular constant matrix, then the columns of $R N(\lambda)$ form a minimal basis with the same column degrees as $N(\lambda)$. 
(b) If $N\left(\lambda_{0}\right)$ has full column rank for all $\lambda_{0} \in \overline{\mathbb{F}}$, then there exists a matrix polynomial $Z(\lambda)$ such that $[N(\lambda) Z(\lambda)]$ is unimodular.

(c) If $N\left(\lambda_{0}\right)$ has full column rank for all $\lambda_{0} \in \mathbb{F}$ and $P(\lambda)$ is any other matrix polynomial such that the product $N(\lambda) P(\lambda)$ is defined, then the invariant polynomials of $P(\lambda)$ and $N(\lambda) P(\lambda)$ are identical.

Results analogous to those in (a), (b), and (c) hold for rows.

Proof. Part (a) follows from Theorem 2.14 and the observation that $R N_{h c}$ is the highest-column-degree coefficient matrix of $R N(\lambda)$.

(b) Let $n \times r, n \geq r$, be the size of $N(\lambda)$, and observe that the Smith form of $N(\lambda)$ is the constant matrix $D=\left[\begin{array}{ll}I_{r} & 0\end{array}\right]^{T} \in \mathbb{F}^{n \times r}$. Therefore, by $(2.1), N(\lambda)=$ $U(\lambda) D V(\lambda)$, with $U(\lambda)$ and $V(\lambda)$ unimodular matrix polynomials, and $N(\lambda)=$ $U(\lambda) \operatorname{diag}\left(V(\lambda), I_{n-r}\right) D$. Finally, define $[N(\lambda) \quad Z(\lambda)]:=U(\lambda) \operatorname{diag}\left(V(\lambda), I_{n-r}\right)$ and note that this matrix is unimodular.

(c) Assume that $N(\lambda) \in \mathbb{F}^{n \times r}$ and $P(\lambda) \in \mathbb{F}^{r \times t}$, and let $P(\lambda)=Q(\lambda) D(\lambda) Y(\lambda)$ be such that $D(\lambda)$ is the Smith form of $P(\lambda)$, and $Q(\lambda)$ and $Y(\lambda)$ are unimodular. Then, by part (b), there exists $Z(\lambda)$ such that $[N(\lambda) Z(\lambda)]$ is unimodular and

$$
N(\lambda) P(\lambda)=[N(\lambda) \quad Z(\lambda)]\left[\begin{array}{ll}
Q(\lambda) & \\
& I_{n-r}
\end{array}\right]\left[\begin{array}{c}
D(\lambda) \\
0_{(n-r) \times t}
\end{array}\right] Y(\lambda)
$$

which proves the result, since $[N(\lambda) \quad Z(\lambda)] \operatorname{diag}\left(Q(\lambda), I_{n-r}\right)$ is unimodular.

We close this section by introducing the eigenstructure of a matrix polynomial.

Definition 2.17. Given an $m \times n$ matrix polynomial $P(\lambda)$ with rank $r$, the eigenstructure of $P(\lambda)$ consists of the following lists of scalar polynomials and nonnegative integers:

(i) the invariant polynomials $p_{1}(\lambda), \ldots, p_{r}(\lambda)$, with degrees $\delta_{1}, \ldots, \delta_{r}$ (finite structure),

(ii) the partial multiplicity sequence at $\infty, \gamma_{1}, \ldots, \gamma_{r}$ (infinite structure),

(iii) the right minimal indices $\varepsilon_{1}, \ldots, \varepsilon_{n-r}$ (right singular structure), and

(iv) the left minimal indices $\eta_{1}, \ldots, \eta_{m-r}$ (left singular structure).

We emphasize that some of the integers in Definition 2.17 can be zero and/or can be repeated. Even all integers in some of the lists (i)-(iv) can be zero. In some recent references (see [10]) the eigenstructure of $P(\lambda)$ is defined to consist only of the lists in parts (i) and (ii) of Definition 2.17, while the name "singular structure" is used for the right and left minimal indices. We are extending here to matrix polynomials the shorter and classical terminology of "eigenstructure" often used for matrix pencils [34]. Note that the name "eigenstructure" of a matrix polynomial has also been used in a somewhat different sense in [35], where the authors include the structural indices at $\infty$ (see Remark 2.8), instead of the partial multiplicity sequence at $\infty$.

3. Matrix polynomials of given degree with prescribed eigenstructure. We first recall the index sum theorem. This theorem establishes a simple relationship between the eigenstructure, the degree, and the rank of any matrix polynomial with coefficients over an arbitrary field. To our knowledge, it was first stated in [25, 28] for matrix polynomials over the real field and was recently extended in [10] to matrix polynomials with coefficients over arbitrary fields.

Theorem 3.1 (index sum theorem). Let $P(\lambda)$ be an $m \times n$ matrix polynomial of degree $d$ and rank $r$ having the following eigenstructure:

- $r$ invariant polynomials $p_{j}(\lambda)$ of degrees $\delta_{j}$, for $j=1, \ldots, r$,

- $r$ infinite partial multiplicities $\gamma_{1}, \ldots, \gamma_{r}$, 
- $n-r$ right minimal indices $\varepsilon_{1}, \ldots, \varepsilon_{n-r}$, and

- $m-r$ left minimal indices $\eta_{1}, \ldots, \eta_{m-r}$,

where some of the degrees, partial multiplicities, or indices can be zero, and/or one or both of the lists of minimal indices can be empty. Then

$$
\sum_{j=1}^{r} \delta_{j}+\sum_{j=1}^{r} \gamma_{j}+\sum_{j=1}^{n-r} \varepsilon_{j}+\sum_{j=1}^{m-r} \eta_{j}=d r .
$$

Remark 3.2. A very interesting remark pointed out by an anonymous referee is that the index sum theorem for matrix polynomials can be obtained as an easy corollary of a more general result valid for arbitrary rational matrices, which is much older than reference [28]. This result is [36, Theorem 3], which can also be found in [18, Theorem 6.5-11]. Using the notion of structural indices at $\alpha$ introduced in Remark 2.8 and [18, p. 452, eq. (32), p. 460, eq. (40)], Theorem 6.5-11 in [18] can be stated as follows: Let $R(\lambda)$ be any $m \times n$ rational matrix with rank $r$, let $\mathcal{S}$ be the set of (different) zeros and poles of $R(\lambda)$ including possibly $\infty$, and let $\eta_{1}, \ldots, \eta_{m-r}$ and $\varepsilon_{1}, \ldots, \varepsilon_{n-r}$ be the left and right minimal indices of $R(\lambda)$, respectively. Then

$$
\sum_{\alpha \in \mathcal{S}}\left(\sum_{i=1}^{r} \sigma_{i}(\alpha)\right)+\sum_{j=1}^{n-r} \varepsilon_{j}+\sum_{j=1}^{m-r} \eta_{j}=0 .
$$

This equation can be applied, in particular, to any $m \times n$ matrix polynomial $P(\lambda)$ of degree $d$, which always has a pole at $\infty$ and so (nonzero) structural indices at $\infty$. In addition, we proved in Remark 2.8 that for $P(\lambda)$ the terms in the sequence $d+\sigma_{1}(\infty) \leq \cdots \leq d+\sigma_{r}(\infty)$ either are all zero or are the $r$ partial multiplicities at $\infty$ of $P(\lambda)$. So, adding $d r$ to both sides of (3.2) applied to $P(\lambda)$, and taking into account the discussion in Remark 2.8 on finite eigenvalues, gives (3.1) and proves the index sum theorem.

The proof of Theorem 3.1 in [10] uses a particular linearization of $P(\lambda)$, together with the corresponding result for pencils. For matrix pencils (i.e., when $d=1$ ), Theorem 3.1 follows from the Kronecker canonical form (KCF) [13], and using this canonical form, one can show also that there exist $m \times n$ matrix pencils with given rank $r$ having all possible regular and singular eigenstructures satisfying the constraint (3.1). However, a similar result for matrix polynomials of degree $d>1$ is not available in the literature and it is not obvious to prove, since there is nothing similar to the KCF for polynomials of degree larger than one. ${ }^{1}$ To establish this result is the main contribution of the present paper. This is presented in Theorem 3.3.

TheOREM 3.3. Let $m, n, d$, and $r \leq \min \{m, n\}$ be given positive integers. Let $p_{1}(\lambda), \ldots, p_{r}(\lambda)$ be $r$ arbitrary monic polynomials with coefficients in an infinite field $\mathbb{F}$ and with respective degrees $\delta_{1}, \ldots, \delta_{r}$ and such that $p_{j}(\lambda)$ divides $p_{j+1}(\lambda)$ for $j=1, \ldots, r-1$. Let $0 \leq \gamma_{1} \leq \cdots \leq \gamma_{r}, 0 \leq \varepsilon_{1} \leq \cdots \leq \varepsilon_{n-r}$, and $0 \leq \eta_{1} \leq \cdots \leq \eta_{m-r}$ be given lists of nonnegative integers. Then, there exists an $m \times n$ matrix polynomial $P(\lambda)$ with coefficients in $\mathbb{F}$, with rank $r$, with degree $d$, with invariant polynomials $p_{1}(\lambda), \ldots, p_{r}(\lambda)$, with partial multiplicities at $\infty$ equal to $\gamma_{1}, \ldots, \gamma_{r}$, and with right and left minimal indices respectively equal to $\varepsilon_{1}, \ldots \varepsilon_{n-r}$ and $\eta_{1}, \ldots, \eta_{m-r}$ if and only if (3.1) holds and $\gamma_{1}=0$.

\footnotetext{
${ }^{1}$ It is interesting to mention here the significative advances on Kronecker-like quasi-canonical forms for quadratic matrix polynomials obtained in [20].
} 
The necessity of (3.1) and $\gamma_{1}=0$ in Theorem 3.3 follows immediately from the index sum theorem and Lemma 2.7. However, the proof of the sufficiency of these conditions is nontrivial and requires a number of auxiliary lemmas, which will be presented in subsection 3.1. This proof is based on techniques that were used in [17, Theorem 5.2] to prove the particular case of Theorem 3.3 in which $P(\lambda)$ has full row or full column rank. We will see that to prove the general case demands considerable more effort.

In subsection 3.2, we present an alternative statement of Theorem 3.3 which is more convenient for proving the results on $\ell$-ifications included in section 4 .

3.1. Auxiliary lemmas and proof of Theorem 3.3. Lemma 3.4 will allow us to reduce the proof of Theorem 3.3 to the case when all partial multiplicities at $\infty$ are zero, i.e., when there are no infinite eigenvalues. Lemma 3.4 is a particular case of [22, Proposition 3.29, Theorems 5.3 and 7.5] or [27, Theorem 4.1]. The statement of Lemma 3.4 does not follow the style of the results in [22], but it is stated exactly as it will be used in the proof of Theorem 3.3.

Lemma 3.4. Let $\mathbb{F}$ be an infinite field, let $\left\{\lambda_{1}, \ldots, \lambda_{s}\right\} \subset \overline{\mathbb{F}}$ be any finite subset of $\overline{\mathbb{F}}$, with $\lambda_{i} \neq \lambda_{j}$ if $i \neq j$, and let $\omega \in \mathbb{F}$ be such that $\omega \neq \lambda_{i}$, for $i=1, \ldots, s$. Let $P_{\omega}(\lambda)$ be an $m \times n$ matrix polynomial with coefficients in $\mathbb{F}$, of degree $d$, and rank $r$. Suppose that $P_{\omega}(\lambda)$ has no infinite eigenvalues, that $1 /\left(\lambda_{1}-\omega\right), \ldots, 1 /\left(\lambda_{s}-\omega\right)$ are the nonzero finite eigenvalues of $P_{\omega}(\lambda)$, and that $\lambda_{0}=0$ is an eigenvalue of $P_{\omega}(\lambda)$ with less than $r$ associated elementary divisors. Let us define the following $m \times n$ matrix polynomial over $\mathbb{F}$ :

$$
P(\lambda):=(\lambda-\omega)^{d} P_{\omega}\left(\frac{1}{\lambda-\omega}\right)
$$

Then

(a) $P(\lambda)$ has degree $d$ and rank $r$;

(b) $\mu_{0}=\infty$ is an eigenvalue of $P(\lambda)$ with partial multiplicity sequence equal to the partial multiplicity sequence of $\lambda_{0}=0$ in $P_{\omega}(\lambda)$;

(c) the finite eigenvalues of $P(\lambda)$ are $\lambda_{1}, \ldots, \lambda_{s}$ and, for each $j=1, \ldots, s$, the partial multiplicity sequence of $\lambda_{j}$ is equal to the partial multiplicity sequence of $1 /\left(\lambda_{j}-\omega\right)$ in $P_{\omega}(\lambda)$;

(d) the minimal indices of $P(\lambda)$ and $P_{\omega}(\lambda)$ are identical.

Proof. As a consequence of the results in $[22,27]$ mentioned above, we only need to prove that $P(\lambda)$ has the same degree as $P_{\omega}(\lambda)$, since Möbius transformations of matrix polynomials do not preserve the degree in general $[22$, p. 6]. In our case, if $P_{\omega}(\lambda)=Q_{0}+\lambda Q_{1}+\cdots+\lambda^{d} Q_{d}$, with $Q_{d} \neq 0$, then $Q_{0} \neq 0$ because $P_{\omega}(\lambda)$ has less than $r$ elementary divisors at 0 . Since

$$
P(\lambda)=(\lambda-\omega)^{d} Q_{0}+(\lambda-\omega)^{d-1} Q_{1}+\cdots+Q_{d},
$$

we obtain that $P(\lambda)$ has degree $d$.

Remark 3.5. Lemma 3.4 is valid only for infinite fields and this is the reason why Theorem 3.3 is valid only for infinite fields as well. In the proof of Theorem 3.3, for proving the existence of a polynomial $P(\lambda)$ with infinite eigenvalues and prescribed complete eigenstructure, in particular with prescribed finite eigenvalues $\lambda_{1}, \ldots, \lambda_{s}$, we will prove instead the existence of a polynomial $P_{\omega}(\lambda)$ as the one in Lemma 3.4 and then the desired $P(\lambda)$ will be given by (3.3). The problem is that if $\mathbb{F}$ is finite, then $\lambda_{1}, \ldots, \lambda_{s}$ might be all the elements in the field and, in this case, we cannot choose $\omega$ as in Lemma 3.4 .

Copyright $@$ by SIAM. Unauthorized reproduction of this article is prohibited. 
Lemma 3.6 will also be used in the proof of Theorem 3.3, more precisely in the proof of the key Lemma 3.8. We do not claim any originality in Lemma 3.6: Lemma 3.6(a) follows easily from techniques in [18, Chapter 6] and Lemma 3.6(b) is explicitly stated in a somewhat different form in [12, p. 503]. However, taking into account the fundamental role that Lemma 3.6 plays in this work, we include proofs of both parts that use only basic results on matrix polynomials, in such a way that any reader can follow them without effort. As we comment below some steps of these proofs follow also from well-known results in multivariable system theory [29], but to understand them requires familiarity with the literature in that area. Note that the convention introduced in Remark 2.15 is used in Lemma 3.6 and, also, very often in this section.

Lemma 3.6. Let $M(\lambda)$ and $N(\lambda)$ be matrix polynomials of sizes $n \times r$ and $n \times$ $(n-r)$, respectively, such that

$$
M(\lambda)^{T} N(\lambda)=0,
$$

and let $M_{h c}$ and $N_{h c}$ be the highest-column-degree coefficient matrices of $M(\lambda)$ and $N(\lambda)$, respectively. Then,

(a) if $M(\lambda)$ and $N(\lambda)$ are both column reduced, then there exists a nonsingular $n \times n$ constant matrix $R$ such that

$$
M_{h c}^{T} R^{-1}=\left[\begin{array}{ll}
0_{r \times(n-r)} & I_{r}
\end{array}\right] \quad \text { and } \quad R N_{h c}=\left[\begin{array}{c}
I_{n-r} \\
0_{r \times(n-r)}
\end{array}\right] ;
$$

(b) if $M(\lambda)$ and $N(\lambda)$ are both minimal bases with column degrees $\widehat{\varepsilon}_{1}, \ldots, \widehat{\varepsilon}_{r}$ and $\varepsilon_{1}, \ldots, \varepsilon_{n-r}$, respectively, then

$$
\sum_{i=1}^{r} \widehat{\varepsilon}_{i}=\sum_{j=1}^{n-r} \varepsilon_{j} .
$$

Proof. (a) Let $\varepsilon_{1}, \ldots, \varepsilon_{n-r}$ be the degrees of the columns of $N(\lambda)$ and let $\widehat{\varepsilon}_{1}, \ldots, \widehat{\varepsilon}_{r}$ be the degrees of the columns of $M(\lambda)$. Define $D_{\varepsilon}(\lambda):=\operatorname{diag}\left(\lambda^{\varepsilon_{1}}, \ldots, \lambda^{\varepsilon_{n-r}}\right)$ and $\widehat{D}_{\varepsilon}(\lambda):=\operatorname{diag}\left(\lambda^{\widehat{\varepsilon}_{1}}, \ldots, \lambda^{\widehat{\varepsilon}_{r}}\right)$. Then, we can write

$$
M(\lambda)=M_{h c} \widehat{D}_{\varepsilon}(\lambda)+\widetilde{M}(\lambda) \quad \text { and } \quad N(\lambda)=N_{h c} D_{\varepsilon}(\lambda)+\widetilde{N}(\lambda) .
$$

Observe that

$$
\operatorname{deg}\left(\operatorname{col}_{j}(\widetilde{M}(\lambda))\right)<\widehat{\varepsilon}_{j}, \text { for } j=1, \ldots, r, \quad \text { and } \operatorname{deg}\left(\operatorname{col}_{j}(\widetilde{N}(\lambda))\right)<\varepsilon_{j},
$$

for $j=1, \ldots, n-r$, where $\operatorname{col}_{j}(A)$ denotes the $j$ th column of a matrix $A$. Since $N_{h c}$ has full column rank, there exists a constant nonsingular $n \times n$ matrix $R_{1}$ such that

$$
R_{1} N_{h c}=\left[\begin{array}{c}
I_{n-r} \\
0_{r \times(n-r)}
\end{array}\right] \quad \text { so } \quad R_{1} N(\lambda)=\left[\begin{array}{c}
I_{n-r} \\
0_{r \times(n-r)}
\end{array}\right] D_{\varepsilon}(\lambda)+R_{1} \tilde{N}(\lambda) .
$$

Therefore, we have $M(\lambda)^{T} R_{1}^{-1} R_{1} N(\lambda)=0$, from (3.4), which, combined with (3.5), implies

$$
\begin{aligned}
\widehat{D}_{\varepsilon}(\lambda) M_{h c}^{T} R_{1}^{-1} & {\left[\begin{array}{c}
I_{n-r} \\
0_{r \times(n-r)}
\end{array}\right] D_{\varepsilon}(\lambda)+\widehat{D}_{\varepsilon}(\lambda) M_{h c}^{T} \widetilde{N}(\lambda) } \\
& +\widetilde{M}(\lambda)^{T} N_{h c} D_{\varepsilon}(\lambda)+\widetilde{M}(\lambda)^{T} \widetilde{N}(\lambda)=0 .
\end{aligned}
$$

Copyright () by SIAM. Unauthorized reproduction of this article is prohibited. 
The right- and left-hand sides of (3.7) are $r \times(n-r)$ matrix polynomials and the $(i, j)$ entry of $(3.7)$ is

$$
\left(M_{h c}^{T} R_{1}^{-1}\left[\begin{array}{c}
I_{n-r} \\
0_{r \times(n-r)}
\end{array}\right]\right)_{i j} \lambda^{\widehat{\varepsilon}_{i}+\varepsilon_{j}}+h_{i j}(\lambda)=0,
$$

where $\operatorname{deg}\left(h_{i j}(\lambda)\right)<\widehat{\varepsilon}_{i}+\varepsilon_{j}$ by $(3.6)$, for $i=1, \ldots, r$ and $j=1, \ldots, n-r$. Therefore,

$$
M_{h c}^{T} R_{1}^{-1}\left[\begin{array}{c}
I_{n-r} \\
0_{r \times(n-r)}
\end{array}\right]=0, \quad \text { which implies } \quad M_{h c}^{T} R_{1}^{-1}=\left[\begin{array}{ll}
0_{r \times(n-r)} & X
\end{array}\right],
$$

where $X$ is an $r \times r$ nonsingular matrix, since $M_{h c}$ has full column rank. Finally, define the $n \times n$ matrix $R_{2}=\operatorname{diag}\left(I_{n-r}, X\right)$, note that

$$
M_{h c}^{T} R_{1}^{-1} R_{2}^{-1}=\left[\begin{array}{ll}
0_{r \times(n-r)} & I_{r}
\end{array}\right] \quad \text { and } \quad R_{2} R_{1} N_{h c}=\left[\begin{array}{c}
I_{n-r} \\
0_{r \times(n-r)}
\end{array}\right],
$$

and take $R=R_{2} R_{1}$.

(b) Let us partition $M(\lambda)^{T}=\left[M_{1}(\lambda)^{T}, M_{2}(\lambda)^{T}\right]$, with $M_{2}(\lambda) \in \mathbb{F}[\lambda]^{r \times r}$, and $N(\lambda)=\left[N_{1}(\lambda)^{T}, N_{2}(\lambda)^{T}\right]^{T}$, with $N_{1}(\lambda) \in \mathbb{F}[\lambda]^{(n-r) \times(n-r)}$. Theorem 2.14 and Lemma 2.16(b) guarantee that $M(\lambda)^{T}$ can be completed to a unimodular matrix

$$
\left[\begin{array}{cc}
U_{11}(\lambda) & U_{12}(\lambda) \\
M_{1}(\lambda)^{T} & M_{2}(\lambda)^{T}
\end{array}\right] \in \mathbb{F}[\lambda]^{n \times n}
$$

Note that

$$
\left[\begin{array}{cc}
U_{11}(\lambda) & U_{12}(\lambda) \\
M_{1}(\lambda)^{T} & M_{2}(\lambda)^{T}
\end{array}\right]\left[\begin{array}{c}
N_{1}(\lambda) \\
N_{2}(\lambda)
\end{array}\right]=\left[\begin{array}{c}
Z(\lambda) \\
0_{r \times(n-r)}
\end{array}\right]
$$

From Theorem 2.14, we get that $\left[I_{n-r}, 0_{(n-r) \times r}\right]^{T}$ is the Smith normal form of $N(\lambda)$, which implies that the matrix $Z(\lambda) \in \mathbb{F}[\lambda]^{(n-r) \times(n-r)}$ in (3.9) is unimodular. Let $R \in \mathbb{F}^{n \times n}$ be a nonsingular constant matrix as in part (a) and define

$$
\begin{aligned}
& {\left[\begin{array}{cc}
\widehat{U}_{11}(\lambda) & \widehat{U}_{12}(\lambda) \\
\widehat{M}_{1}(\lambda)^{T} & \widehat{M}_{2}(\lambda)^{T}
\end{array}\right]:=\left[\begin{array}{cc}
U_{11}(\lambda) & U_{12}(\lambda) \\
M_{1}(\lambda)^{T} & M_{2}(\lambda)^{T}
\end{array}\right] R^{-1} \quad \text { and } } \\
& {\left[\begin{array}{l}
\widehat{N}_{1}(\lambda) \\
\widehat{N}_{2}(\lambda)
\end{array}\right]:=R\left[\begin{array}{l}
N_{1}(\lambda) \\
N_{2}(\lambda)
\end{array}\right] }
\end{aligned}
$$

where, as a consequence of part (a), both $\widehat{M}_{2}(\lambda)$ and $\widehat{N}_{1}(\lambda)$ are square nonsingular matrix polynomials with highest-column-degree coefficient matrices equal to $I_{r}$ and $I_{n-r}$, respectively. ${ }^{2}$ Next consider the matrix polynomial

$$
V(\lambda):=\left[\begin{array}{ll}
V_{11}(\lambda) & V_{12}(\lambda) \\
V_{21}(\lambda) & V_{22}(\lambda)
\end{array}\right]:=\left[\begin{array}{cc}
\widehat{U}_{11}(\lambda) & \widehat{U}_{12}(\lambda) \\
\widehat{M}_{1}(\lambda)^{T} & \widehat{M}_{2}(\lambda)^{T}
\end{array}\right]^{-1}
$$

\footnotetext{
${ }^{2}$ At this point, a reader familiar with control theory can proceed via the following argument, which has been kindly provided by an anonymous referee. The definitions of the concepts mentioned in this argument can be found in [18, 29]. Since $\widehat{M}_{1}(\lambda)^{T} \widehat{N}_{1}(\lambda)+\widehat{M}_{2}(\lambda)^{T} \widehat{N}_{2}(\lambda)=0$, we get $\widehat{M}_{2}(\lambda)^{-T} \widehat{M}_{1}(\lambda)^{T}=-\widehat{N}_{2}(\lambda) \widehat{N}_{1}(\lambda)^{-1}$. Moreover, $\widehat{M}_{1}(\lambda)^{T}, \widehat{M}_{2}(\lambda)^{T}$ are left-coprime and $\widehat{N}_{1}(\lambda), \widehat{N}_{2}(\lambda)$ are right-coprime. So, $\widehat{M}_{2}(\lambda)^{-T} \widehat{M}_{1}(\lambda)^{T}$ and $\widehat{N}_{2}(\lambda) \widehat{N}_{1}(\lambda)^{-1}$ are two irreducible matrix-fraction descriptions of the same transfer matrix function. Then, it follows from [29, Theorem 3.1, Chapter 3] that $\widehat{M}_{2}(\lambda)$ and $\widehat{N}_{1}(\lambda)$ have the same nontrivial invariant polynomials and so the same determinant up to a product by a nonzero constant. This latter determinantal property is what we need to continue the proof and what we prove above by other means.
}

Copyright @ $\odot$ by SIAM. Unauthorized reproduction of this article is prohibited. 
and combine this definition with (3.9) and (3.10) to get

$$
\left[\begin{array}{l}
\widehat{N}_{1}(\lambda) \\
\widehat{N}_{2}(\lambda)
\end{array}\right]=\left[\begin{array}{ll}
V_{11}(\lambda) & V_{12}(\lambda) \\
V_{21}(\lambda) & V_{22}(\lambda)
\end{array}\right]\left[\begin{array}{c}
Z(\lambda) \\
0
\end{array}\right]
$$

which implies $\widehat{N}_{1}(\lambda)=V_{11}(\lambda) Z(\lambda)$ and, since $Z(\lambda)$ is unimodular,

$$
\operatorname{deg}\left(\operatorname{det}\left(\widehat{N}_{1}(\lambda)\right)\right)=\operatorname{deg}\left(\operatorname{det}\left(V_{11}(\lambda)\right)\right) .
$$

Next, since the matrices in (3.11) are unimodular, a standard property of minors of inverses [16, p. 21] implies that $\operatorname{det}\left(V_{11}(\lambda)\right) / \operatorname{det}\left(\widehat{M}_{2}(\lambda)\right)=\operatorname{det}(V(\lambda))$ is a nonzero constant, which combined with (3.12) yields

$$
\operatorname{deg}\left(\operatorname{det}\left(\widehat{M}_{2}(\lambda)\right)\right)=\operatorname{deg}\left(\operatorname{det}\left(\widehat{N}_{1}(\lambda)\right)\right) .
$$

Since the highest-column-degree coefficient matrices of $\widehat{M}_{2}(\lambda)$ and $\widehat{N}_{1}(\lambda)$ are $I_{r}$ and $I_{n-r}$, respectively, we get, taking into account (3.10), that

$$
\sum_{i=1}^{r} \widehat{\varepsilon}_{i}=\operatorname{deg}\left(\operatorname{det}\left(\widehat{M}_{2}(\lambda)\right)\right)=\operatorname{deg}\left(\operatorname{det}\left(\widehat{N}_{1}(\lambda)\right)\right)=\sum_{j=1}^{n-r} \varepsilon_{j} .
$$

Lemma 3.7 is a simple technical result needed to prove Lemma 3.8. Observe that Lemma 3.7 is nothing but [29, Lemma 4.1, Chapter 5] with the unimodular transformations written explicitly in a way that is convenient for their use in this paper.

Lemma 3.7. Let $k$ and $d$ be nonnegative integers such that $k-d>1$. Let $Q(\lambda)$ be a $2 \times 2$ matrix polynomial

$$
Q(\lambda)=\left[\begin{array}{ll}
a(\lambda) & b(\lambda) \\
c(\lambda) & e(\lambda)
\end{array}\right]
$$

such that

$$
\operatorname{deg}(a(\lambda))=d, \quad \operatorname{deg}(b(\lambda)) \leq d-1, \quad \operatorname{deg}(c(\lambda)) \leq k-1, \quad \operatorname{deg}(e(\lambda))=k,
$$

and $a(\lambda)$ and $e(\lambda)$ are monic. Let $c(\lambda)=c_{k-1} \lambda^{k-1}+c_{k-2} \lambda^{k-2}+\cdots+c_{0}$ and

$$
\left[\begin{array}{ll}
\widetilde{a}(\lambda) & \widetilde{b}(\lambda) \\
\widetilde{c}(\lambda) & \widetilde{e}(\lambda)
\end{array}\right]:=\left[\begin{array}{cc}
1 & 0 \\
\left(c_{k-1}+1\right) \lambda^{k-d-1} & -1
\end{array}\right] Q(\lambda)\left[\begin{array}{ll}
1 & \lambda \\
0 & 1
\end{array}\right] .
$$

Then,

$$
\operatorname{deg}(\widetilde{a}(\lambda))=d, \quad \operatorname{deg}(\widetilde{b}(\lambda))=d+1, \quad \operatorname{deg}(\widetilde{c}(\lambda))=k-1, \quad \operatorname{deg}(\widetilde{e}(\lambda)) \leq k-1,
$$

and $\widetilde{a}(\lambda), \widetilde{b}(\lambda)$, and $\widetilde{c}(\lambda)$ are monic.

Proof. A direct computation yields

$$
\begin{aligned}
& {\left[\begin{array}{cc}
\left(c_{k-1}+1\right) \lambda^{k-d-1} & 0
\end{array}\right] Q(\lambda)\left[\begin{array}{cc}
1 & \lambda \\
0 & 1
\end{array}\right]} \\
& =\left[\begin{array}{c|c}
a(\lambda) & \lambda a(\lambda)+b(\lambda) \\
\hline\left(c_{k-1}+1\right) \lambda^{k-d-1} a(\lambda)-c(\lambda) & \left(c_{k-1}+1\right) \lambda^{k-d-1}(\lambda a(\lambda)+b(\lambda))-(\lambda c(\lambda)+e(\lambda))
\end{array}\right] .
\end{aligned}
$$

Copyright $\odot$ by SIAM. Unauthorized reproduction of this article is prohibited. 
The result follows immediately from this expression.

Lemma 3.8 shows that given any lists of invariant polynomials and right minimal indices there always exists a row reduced matrix polynomial $P(\lambda)$ with the degrees of its rows "as close as possible" and having precisely these invariant polynomials and right minimal indices. Observe that such $P(\lambda)$ has full row rank, i.e., it has no left minimal indices, since it is row reduced, and if its row degrees were all equal, then $P(\lambda)$ would not have infinite eigenvalues. Therefore, by (3.1), the row degrees of $P(\lambda)$ can all be equal only if the sum of the degrees of the given invariant polynomials plus the given right minimal indices is a multiple of the number of invariant polynomials. Lemma 3.8 is the key piece in the proof of Theorem 3.3 and its proof is rather technical.

LEMMA 3.8. Let $r, n$ be two positive integers with $r \leq n$. Let $p_{1}(\lambda), \ldots, p_{r}(\lambda)$ be $r$ monic scalar polynomials, with respective degrees $\delta_{1} \leq \cdots \leq \delta_{r}$ and such that $p_{j}(\lambda)$ is a divisor of $p_{j+1}(\lambda)$ for $j=1, \ldots, r-1$. Let $\varepsilon_{1} \leq \cdots \leq \varepsilon_{n-r}$ be a list of $n-r$ nonnegative integers ( which is empty if $n=r$ ). Define

$$
\delta:=\sum_{j=1}^{r} \delta_{j} \quad \text { and } \quad \varepsilon:=\sum_{j=1}^{n-r} \varepsilon_{j}
$$

and write

$$
\delta+\varepsilon=r q_{\varepsilon}+t_{\varepsilon}, \quad \text { where } \quad 0 \leq t_{\varepsilon}<r .
$$

Then there exists an $r \times n$ row reduced matrix polynomial $P(\lambda)$ with $t_{\varepsilon}$ row degrees equal to $q_{\varepsilon}+1$ and $r-t_{\varepsilon}$ row degrees equal to $q_{\varepsilon}$, and such that $p_{1}(\lambda), \ldots, p_{r}(\lambda)$ and $\varepsilon_{1}, \ldots, \varepsilon_{n-r}$ are, respectively, the invariant polynomials and the right minimal indices of $P(\lambda)$. The degree of $P(\lambda)$ is thus $q_{\varepsilon}+1$ if $t_{\varepsilon}>0$ and $q_{\varepsilon}$ otherwise.

Proof. The proof closely follows the proof of Theorem 5.2 in [17]. We prove first in detail the most difficult case $r<n$ and sketch at the end the proof of the much easier case $r=n$. Let $\widetilde{N}(\lambda) \in \mathbb{F}[\lambda]^{n \times(n-r)}$ be any (right) minimal basis with column degrees $\varepsilon_{1} \leq \cdots \leq \varepsilon_{n-r}$. A specific way to construct such $\tilde{N}(\lambda)$ is to place on the main diagonal $\lambda^{\varepsilon_{1}}, \ldots, \lambda^{\varepsilon_{n-r}}$ and on the first subdiagonal $1, \ldots, 1$. Now, let $\widetilde{Q}(\lambda) \in \mathbb{F}[\lambda]^{r \times n}$ be any minimal basis of $\mathcal{N}_{\ell}(\widetilde{N})$ and let $\widehat{\varepsilon}_{1} \leq \cdots \leq \widehat{\varepsilon}_{r}$ be its row degrees. So, $\widetilde{Q}(\lambda) \widetilde{N}(\lambda)=0$ and according to Lemma 3.6(b),

$$
\varepsilon=\sum_{i=1}^{r} \widehat{\varepsilon}_{i}=\sum_{j=1}^{n-r} \varepsilon_{j} .
$$

Next, define $\widehat{Q}(\lambda):=\operatorname{diag}\left(p_{1}(\lambda), \ldots, p_{r}(\lambda)\right) \widetilde{Q}(\lambda)$. Observe that $\widehat{Q}(\lambda)$ is row reduced, since $\widetilde{Q}(\lambda)$ is, and $\widehat{Q}_{h r}=\widetilde{Q}_{h r}$, i.e., the highest-row-degree coefficient matrices are equal, $\widehat{Q}(\lambda) \widetilde{N}(\lambda)=0$, the row degrees of $\widehat{Q}(\lambda)$ are $\widehat{\varepsilon}_{1}+\delta_{1} \leq \cdots \leq \widehat{\varepsilon}_{r}+\delta_{r}$, and $p_{1}(\lambda), \ldots, p_{r}(\lambda)$ are the invariant polynomials of $\widehat{Q}(\lambda)$, by Lemma 2.16(c) (for rows). Therefore, we can apply Lemma 3.6(a) to $\widehat{Q}(\lambda) \widetilde{N}(\lambda)=0$ and to prove the existence of a nonsingular matrix $R$ with the properties specified in that lemma. This matrix $R$ allows us to define the following two matrix polynomials:

$$
Q(\lambda)=\widehat{Q}(\lambda) R^{-1} \in \mathbb{F}[\lambda]^{r \times n} \quad \text { and } \quad N(\lambda)=R \tilde{N}(\lambda) \in \mathbb{F}[\lambda]^{n \times(n-r)} .
$$

From the discussion above, it is easy to check that $Q(\lambda)$ and $N(\lambda)$ satisfy the following:

(i) $Q(\lambda) N(\lambda)=0$, 
(ii) $Q(\lambda)$ is row reduced and $N(\lambda)$ is a minimal basis of $\mathcal{N}_{r}(Q)$,

(iii) $Q_{h r}=\left[\begin{array}{ll}0_{r \times(n-r)} & I_{r}\end{array}\right]$ and $N_{h c}=\left[\begin{array}{ll}I_{n-r} & 0_{(n-r) \times r}\end{array}\right]^{T}$,

(iv) $Q(\lambda)$ has invariant polynomials $p_{1}(\lambda), \ldots, p_{r}(\lambda)$ and right minimal indices $\varepsilon_{1}, \ldots, \varepsilon_{n-r}$

(v) the sum of the row degrees of $Q(\lambda)$ is $\delta+\varepsilon$.

In addition, the matrix polynomial $Q(\lambda)$ has row degrees $\widehat{\varepsilon}_{1}+\delta_{1} \leq \cdots \leq \widehat{\varepsilon}_{r}+\delta_{r}$, which are denoted by

$$
d_{1}:=\widehat{\varepsilon}_{1}+\delta_{1}, d_{2}:=\widehat{\varepsilon}_{2}+\delta_{2}, \ldots, d_{r-1}:=\widehat{\varepsilon}_{r-1}+\delta_{r-1}, d_{r}:=\widehat{\varepsilon}_{r}+\delta_{r},
$$

for simplicity. So, $Q(\lambda)$ may not have the row degrees of the sought $P(\lambda)$ in the statement of Lemma 3.8, but $Q(\lambda)$ satisfies all the other properties of $P(\lambda)$. Observe, moreover, that the sum of the row degrees of $P(\lambda)$ is also $\delta+\varepsilon$.

If $d_{r}-d_{1} \leq 1$, then the degrees of the $r$ rows of $Q(\lambda)$ differ at most by 1 and their sum is $\delta+\varepsilon$, which implies that the row degrees of $Q(\lambda)$ are the ones in the statement and the proof is finished by taking $P(\lambda)=Q(\lambda)$. Therefore, let us assume $d_{r}-d_{1}>1$ in the rest of the proof. The strategy of the rest of the proof consists in applying very particular unimodular transformations $U(\lambda)=\Pi \widetilde{U}(\lambda)$ and $V(\lambda)$, where $\Pi$ is a permutation,

$$
Q(\lambda) \rightarrow U(\lambda) Q(\lambda) V(\lambda), \quad N(\lambda) \rightarrow V(\lambda)^{-1} N(\lambda)
$$

in such a way that $U(\lambda) Q(\lambda) V(\lambda)$ and $V(\lambda)^{-1} N(\lambda)$ satisfy the properties (i), (ii), (iii), (iv), and (v) above and, in addition, the row degrees of $\widetilde{U}(\lambda) Q(\lambda) V(\lambda)$ are $d_{1}+1, d_{2}, \ldots, d_{r-1}, d_{r}-1$, while $\Pi \widetilde{U}(\lambda) Q(\lambda) V(\lambda)$ has its row degrees in nondecreasing order. Clearly after a finite sequence of this type of transformations we will get a matrix polynomial $Q_{\text {last }}(\lambda)$ which will have the properties of $P(\lambda)$ in the statement.

Let us show how to construct the unimodular matrices $U(\lambda)$ and $V(\lambda)$ mentioned in the previous paragraph. For this purpose let us partition the matrix polynomials $Q(\lambda)$ and $N(\lambda)$ as follows:

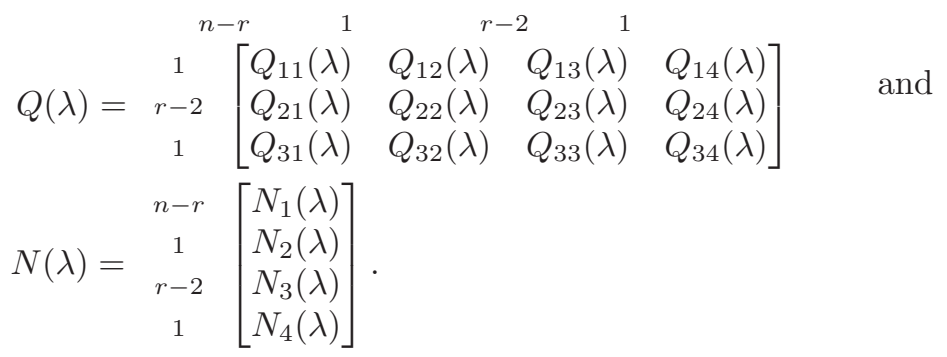

Observe that, as a consequence of property (iii), i.e., of the structure of $Q_{h r}$, and (3.15), $\operatorname{deg}\left(Q_{12}(\lambda)\right)=d_{1}, \operatorname{deg}\left(Q_{14}(\lambda)\right) \leq d_{1}-1, \operatorname{deg}\left(Q_{32}(\lambda)\right) \leq d_{r}-1$, and $\operatorname{deg}\left(Q_{34}(\lambda)\right)=d_{r}$. Therefore, by Lemma 3.7, there exists $\alpha \in \mathbb{F}$ such that

$$
\left[\begin{array}{cc}
1 & 0 \\
\alpha \lambda^{d_{r}-d_{1}-1} & -1
\end{array}\right]\left[\begin{array}{ll}
Q_{12}(\lambda) & Q_{14}(\lambda) \\
Q_{32}(\lambda) & Q_{34}(\lambda)
\end{array}\right]\left[\begin{array}{cc}
1 & \lambda \\
0 & 1
\end{array}\right]=\left[\begin{array}{ll}
J_{12}(\lambda) & J_{14}(\lambda) \\
J_{32}(\lambda) & J_{34}(\lambda)
\end{array}\right]
$$

Copyright () by SIAM. Unauthorized reproduction of this article is prohibited. 
with $\operatorname{deg}\left(J_{12}(\lambda)\right)=d_{1}, \operatorname{deg}\left(J_{14}(\lambda)\right)=d_{1}+1, \operatorname{deg}\left(J_{32}(\lambda)\right)=d_{r}-1$, and $\operatorname{deg}\left(J_{34}(\lambda)\right) \leq$ $d_{r}-1$. Based on (3.18), we define the following unimodular matrices:

$$
\begin{aligned}
\widetilde{U}(\lambda) & :=\left[\begin{array}{ccc}
1 & 0 & 0 \\
0 & I_{r-2} & 0 \\
\alpha \lambda^{d_{r}-d_{1}-1} & 0 & -1
\end{array}\right] \in \mathbb{F}[\lambda]^{r \times r} \text { and } \\
\widetilde{V}(\lambda) & :=\left[\begin{array}{cccc}
I_{n-r} & 0 & 0 & 0 \\
0 & 1 & 0 & \lambda \\
0 & 0 & I_{r-2} & 0 \\
0 & 0 & 0 & 1
\end{array}\right] \in \mathbb{F}[\lambda]^{n \times n} .
\end{aligned}
$$

Direct computations taking into account (3.18) show that

$$
\begin{aligned}
& J(\lambda):=\widetilde{U}(\lambda) Q(\lambda) \widetilde{V}(\lambda) \\
& =\left[\begin{array}{cccc}
Q_{11}(\lambda) & J_{12}(\lambda) & Q_{13}(\lambda) & J_{14}(\lambda) \\
Q_{21}(\lambda) & Q_{22}(\lambda) & Q_{23}(\lambda) & Q_{24}(\lambda)+\lambda Q_{22}(\lambda) \\
\alpha \lambda^{d_{r}-d_{1}-1} Q_{11}(\lambda)-Q_{31}(\lambda) & J_{32}(\lambda) & \alpha \lambda^{d_{r}-d_{1}-1} Q_{13}(\lambda)-Q_{33}(\lambda) & J_{34}(\lambda)
\end{array}\right],
\end{aligned}
$$

where, for $i=1,3$ and $j=2,4$, the $J_{i j}(\lambda)$ blocks are those in (3.18), and also

$$
H(\lambda):=\tilde{V}(\lambda)^{-1} N(\lambda)=\left[\begin{array}{cccc}
I_{n-r} & 0 & 0 & 0 \\
0 & 1 & 0 & -\lambda \\
0 & 0 & I_{r-2} & 0 \\
0 & 0 & 0 & 1
\end{array}\right]\left[\begin{array}{c}
N_{1}(\lambda) \\
N_{2}(\lambda) \\
N_{3}(\lambda) \\
N_{4}(\lambda)
\end{array}\right]=\left[\begin{array}{c}
N_{1}(\lambda) \\
N_{2}(\lambda)-\lambda N_{4}(\lambda) \\
N_{3}(\lambda) \\
N_{4}(\lambda)
\end{array}\right] .
$$

Taking into account the properties (i), (ii), (iii), (iv), and (v) of $Q(\lambda)$ and $N(\lambda)$, (3.15), (3.18), (3.19), and (3.20), we deduce easily the following properties for $J(\lambda)$ and $H(\lambda)$ :

1. $J(\lambda) H(\lambda)=0$.

2. $J(\lambda)$ has the same invariant polynomials as $Q(\lambda)$, that is, $p_{1}(\lambda), \ldots, p_{r}(\lambda)$.

3. The row degrees of $J(\lambda)$ are $d_{1}+1, d_{2}, \ldots, d_{r-1}, d_{r}-1$ and its sum is equal to $\delta+\varepsilon$.

4. $J(\lambda)$ is row reduced, since

$$
J_{h r}=\left[\begin{array}{cccc}
0 & 0 & 0 & 1 \\
0 & 0 & I_{r-2} & \mathrm{X} \\
\mathrm{X} & 1 & \mathrm{X} & \mathrm{X}
\end{array}\right],
$$

where the entries in $\mathrm{X}$ are not specified.

5. The column degrees of $H(\lambda)$ are the same as those of $N(\lambda)$, that is, $\varepsilon_{1}, \ldots, \varepsilon_{n-r}$.

6. $H(\lambda)$ is column reduced since

$$
H_{h c}=\left[\begin{array}{c}
I_{n-r} \\
\mathrm{X} \\
0 \\
0
\end{array}\right] .
$$

7. For all $\lambda_{0} \in \overline{\mathbb{F}}, H\left(\lambda_{0}\right)=\widetilde{V}\left(\lambda_{0}\right)^{-1} N\left(\lambda_{0}\right)$ has full column rank, since $N\left(\lambda_{0}\right)$ has. Therefore, $H(\lambda)$ is a minimal basis of $\mathcal{N}_{r}(J)$ by Theorem 2.14. 
Two additional operations are needed in order to get the unimodular transformations announced in (3.16): first, a permutation $\Pi$ such that the rows of $\Pi J(\lambda)=$ $\Pi \widetilde{U}(\lambda) Q(\lambda) \widetilde{V}(\lambda)$ are sorted with nondecreasing degrees, and second, to use Lemma 3.6 (a) to prove that there exists a nonsingular constant matrix $\widetilde{R}$ such that $\Pi J(\lambda) \widetilde{R}^{-1}=\Pi \widetilde{U}(\lambda) Q(\lambda) \widetilde{V}(\lambda) \widetilde{R}^{-1}$ has highest-row-degree coefficient matrix equal to $\left[\begin{array}{ll}0_{r \times(n-r)} & I_{r}\end{array}\right]$ and $\widetilde{R} H(\lambda)=\widetilde{R} \widetilde{V}(\lambda)^{-1} N(\lambda)$ has highest-column-degree coefficient matrix equal to $\left[\begin{array}{ll}I_{n-r} & 0_{(n-r) \times r}\end{array}\right]^{T}$. Therefore, the matrices $U(\lambda)$ and $V(\lambda)$ in (3.16) are

$$
U(\lambda)=\Pi \widetilde{U}(\lambda) \quad \text { and } \quad V(\lambda)=\widetilde{V}(\lambda) \widetilde{R}^{-1},
$$

and the proof of Lemma 3.8 is completed when $r<n$.

The proof of the case $r=n$ is much easier, since there are no right minimal indices, and we simply apply the unimodular transformations $U(\lambda)$ and $V(\lambda)$ considered above to $Q(\lambda):=\operatorname{diag}\left(p_{1}(\lambda), \ldots, p_{r}(\lambda)\right)$.

If in Lemma 3.8 we take $p_{j}(\lambda)=1$ for $j=1, \ldots r$, and use Theorem 2.14, then we get directly Lemma 3.9 .

Lemma 3.9. Let $r, n$ be two positive integers with $r \leq n$ and let $\varepsilon_{1} \leq \cdots \leq \varepsilon_{n-r}$ be a list of $n-r$ nonnegative integers (which is empty if $n=r$ ). Define

$$
\varepsilon:=\sum_{j=1}^{n-r} \varepsilon_{j}
$$

and write it as

$$
\varepsilon=r q_{\varepsilon}+t_{\varepsilon}, \quad \text { where } 0 \leq t_{\varepsilon}<r .
$$

Then there exists an $r \times n$ row reduced matrix polynomial $P(\lambda)$ with $t_{\varepsilon}$ row degrees equal to $q_{\varepsilon}+1$ and $r-t_{\varepsilon}$ row degrees equal to $q_{\varepsilon}$, and such that all the invariant polynomials of $P(\lambda)$ are trivial and $\varepsilon_{1} \leq \cdots \leq \varepsilon_{n-r}$ are its right minimal indices. The degree of $P(\lambda)$ is thus $q_{\varepsilon}+1$ if $t_{\varepsilon}>0$ and $q_{\varepsilon}$ otherwise. In particular, the rows of $P(\lambda)$ form a minimal basis.

Lemma 3.9 implies just by transposition a similar result for the existence of column reduced matrix polynomials with prescribed left minimal indices. This is Lemma 3.10.

LEMma 3.10. Let $r, m$ be two positive integers with $r \leq m$ and let $\eta_{1} \leq \cdots \leq \eta_{m-r}$ be a list of $m-r$ nonnegative integers (which is empty if $m=r$ ). Define

$$
\eta:=\sum_{j=1}^{m-r} \eta_{j}
$$

and write it as

$$
\eta=r q_{\eta}+t_{\eta}, \quad \text { where } \quad 0 \leq t_{\eta}<r .
$$

Then there exists an $m \times r$ column reduced matrix polynomial $P(\lambda)$ with $t_{\eta}$ column degrees equal to $q_{\eta}+1$ and $r-t_{\eta}$ column degrees equal to $q_{\eta}$, and such that all the invariant polynomials of $P(\lambda)$ are trivial and $\eta_{1} \leq \cdots \leq \eta_{m-r}$ are its left minimal indices. The degree of $P(\lambda)$ is thus $q_{\eta}+1$ if $t_{\eta}>0$ and $q_{\eta}$ otherwise. In particular, the columns of $P(\lambda)$ form a minimal basis.

With Lemmas 3.10 and 3.8 at hand, we are in position to prove the main result in this paper: Theorem 3.3.

Copyright $@$ by SIAM. Unauthorized reproduction of this article is prohibited. 
Proof of Theorem 3.3. As we commented in the paragraph just below the statement of Theorem 3.3 the existence of $P(\lambda)$ implies immediately (3.1) and $\gamma_{1}=0$. So, it remains to prove that (3.1) and $\gamma_{1}=0$ imply that $P(\lambda)$ exists.

In the first place, let us show that if there are nonzero partial multiplicities at $\infty$, then we can reduce the proof to the case in which all partial multiplicities at $\infty$ are zero. For this purpose, let $\left\{\lambda_{1}, \ldots, \lambda_{s}\right\} \subset \overline{\mathbb{F}}$ be the union of the sets of (different) roots of $p_{1}(\lambda), \ldots, p_{r}(\lambda)$, i.e., $\lambda_{i} \neq \lambda_{k}$ if $i \neq k$, and let us express the polynomials $p_{j}(\lambda)$ as

$$
p_{j}(\lambda)=\left(\lambda-\lambda_{1}\right)^{\delta_{j}\left(\lambda_{1}\right)} \cdots\left(\lambda-\lambda_{s}\right)^{\delta_{j}\left(\lambda_{s}\right)}, \quad j=1, \ldots r,
$$

where some $\delta_{j}\left(\lambda_{i}\right)$ may be 0 . Let $\omega \in \mathbb{F}$ be such that $\omega \neq \lambda_{i}$, for $i=1, \ldots s$, and define the polynomials

$$
q_{j}(\lambda)=\lambda^{\gamma_{j}}\left(\lambda-\frac{1}{\lambda_{1}-\omega}\right)^{\delta_{j}\left(\lambda_{1}\right)} \cdots\left(\lambda-\frac{1}{\lambda_{s}-\omega}\right)^{\delta_{j}\left(\lambda_{s}\right)}, \quad j=1, \ldots r,
$$

which satisfy that $q_{j}(\lambda)$ divides $q_{j+1}(\lambda)$ for $j=1, \ldots, r-1$. Note, in addition, that $q_{j}(\lambda)$ has coefficients in $\mathbb{F}$ (not only in $\overline{\mathbb{F}}$ ). This latter property follows from the identity

$$
q_{j}(\lambda)=\lambda^{\operatorname{deg}\left(p_{j}\right)+\gamma_{j}} \frac{p_{j}\left(\omega+\frac{1}{\lambda}\right)}{p_{j}(\omega)}
$$

and the fact that $p_{j}(\lambda) \in \mathbb{F}[\lambda]$. Observe that since $k_{j}:=\operatorname{deg}\left(q_{j}\right)=\operatorname{deg}\left(p_{j}\right)+\gamma_{j}$, we have from (3.1) that

$$
\sum_{j=1}^{r} k_{j}+\sum_{j=1}^{n-r} \varepsilon_{j}+\sum_{j=1}^{m-r} \eta_{j}=d r .
$$

Therefore, if we construct an $m \times n$ matrix polynomial $P_{\omega}(\lambda)$ with coefficients in $\mathbb{F}$, with rank $r$, with degree $d$, with invariant polynomials $q_{1}(\lambda), \ldots, q_{r}(\lambda)$, without eigenvalues at $\infty$, with right minimal indices $\varepsilon_{1}, \ldots, \varepsilon_{n-r}$, and with left minimal indices $\eta_{1}, \ldots, \eta_{m-r}$, then, according to Lemma 3.4 , the polynomial $P(\lambda)$ in (3.3) is the polynomial in Theorem 3.3 (with $\gamma_{1}, \ldots, \gamma_{r}$ partial multiplicities at $\infty$ ). Therefore, in the rest of the proof we assume that $\gamma_{1}=\cdots=\gamma_{r}=0$.

In this scenario, the hypothesis (3.1) becomes $\delta+\varepsilon+\eta=d r$, with $\delta=\sum_{j=1}^{r} \delta_{j}$, $\varepsilon=\sum_{j=1}^{n-r} \varepsilon_{j}$, and $\eta=\sum_{j=1}^{m-r} \eta_{j}$. Let $K(\lambda)$ be an $m \times r$ matrix polynomial with the properties of $P(\lambda)$ in Lemma 3.10 and with columns sorted in nondecreasing order of degrees, i.e., each of the first $r-t_{\eta}$ columns of $K(\lambda)$ has degree $q_{\eta}$ and the remaining ones have degree $q_{\eta}+1$. Let $M(\lambda)$ be an $r \times n$ matrix polynomial with the properties of $P(\lambda)$ in Lemma 3.8 and with rows sorted in nonincreasing order of degrees, i.e., each of the first $t_{\varepsilon}$ rows of $M(\lambda)$ has degree $q_{\varepsilon}+1$ and the remaining ones have degree $q_{\varepsilon}$. Define the $m \times n$ matrix polynomial

$$
P(\lambda)=K(\lambda) M(\lambda) \in \mathbb{F}[\lambda]^{m \times n},
$$

which satisfies the following properties:

1. $\operatorname{rank}(P)=r$, since both $K(\lambda)$ and $M(\lambda)$ have rank $r$.

2. The invariant polynomials of $P(\lambda)$ are identical to the invariant polynomials of $M(\lambda)$, that is, $p_{1}(\lambda), \ldots, p_{r}(\lambda)$, since $K(\lambda)$ is a minimal basis and Lemma 2.16(c) can be applied. 
3. The right minimal indices of $P(\lambda)$ are $\varepsilon_{1}, \ldots, \varepsilon_{n-r}$, since $\mathcal{N}_{r}(P)=\mathcal{N}_{r}(M)$.

4. The left minimal indices of $P(\lambda)$ are $\eta_{1}, \ldots, \eta_{m-r}$, since $\mathcal{N}_{\ell}(P)=\mathcal{N}_{\ell}(K)$. Therefore, it only remains to prove that $\operatorname{deg}(P)=d$ and that $P(\lambda)$ has no eigenvalue at $\infty$.

For this purpose, note that $\eta=r q_{\eta}+t_{\eta}$, with $0 \leq t_{\eta}<r$, and $\delta+\varepsilon=r q_{\varepsilon}+t_{\varepsilon}$, with $0 \leq t_{\varepsilon}<r$, imply $d r=\delta+\varepsilon+\eta=r\left(q_{\eta}+q_{\varepsilon}\right)+\left(t_{\eta}+t_{\varepsilon}\right)$, with $0 \leq t_{\eta}+t_{\varepsilon}<2 r$. But $t_{\eta}+t_{\varepsilon}=r\left(d-q_{\eta}-q_{\varepsilon}\right)$ is a multiple of $r$ and, therefore, $t_{\eta}+t_{\varepsilon}=0$ or $t_{\eta}+t_{\varepsilon}=r$ and

$$
d= \begin{cases}q_{\eta}+q_{\varepsilon} & \text { if } t_{\eta}+t_{\varepsilon}=0 \\ q_{\eta}+q_{\varepsilon}+1 & \text { if } t_{\eta}+t_{\varepsilon}=r .\end{cases}
$$

On the other hand, if $\operatorname{col}_{i}(A)$ (resp., $\operatorname{row}_{i}(A)$ ) denotes the $i$ th column (resp., row) of a matrix $A$, we get from (3.23)

$$
P(\lambda)=\operatorname{col}_{1}(K) \operatorname{row}_{1}(M)+\cdots+\operatorname{col}_{r}(K) \operatorname{row}_{r}(M)
$$

and observe that if $t_{\eta}+t_{\varepsilon}=0$, then all summands in (3.24) have degree $d=q_{\eta}+q_{\varepsilon}$, while if $t_{\eta}+t_{\varepsilon}=r$, then all summands in (3.24) have degree $d=q_{\eta}+q_{\varepsilon}+1$. In both cases the matrix coefficient of degree $d$ in $P(\lambda)$ is

$$
P_{d}=K_{h c} M_{h r},
$$

where $K_{h c}$ is the highest-column-degree coefficient matrix of $K(\lambda)$ and $M_{h r}$ is the highest-row-degree coefficient matrix of $M(\lambda)$. Since $K(\lambda)$ is column reduced and $M(\lambda)$ is row reduced, we get that $\operatorname{rank}\left(P_{d}\right)=r$, which implies that $\operatorname{deg}(P)=d$ and that $P(\lambda)$ has no infinite eigenvalues. This completes the proof of Theorem 3.3.

3.2. Theorem 3.3 expressed in terms of lists of elementary divisors and minimal indices. Theorem 3.12 below expresses Theorem 3.3 as a realizability result in the spirit of results included in [20] for quadratic matrix polynomials or, equivalently, as a general inverse eigenproblem of matrix polynomials. Theorem 3.12 has the advantage of not assuming in advance which are the rank and the size of the polynomial $P(\lambda)$ whose existence is established. This feature is very convenient for proving the results on $\ell$-ifications included in section 4 .

Theorem 3.12 uses the concepts introduced in Definition 3.11.

Definition 3.11. A list $\mathcal{L}$ of elementary divisors over a field $\mathbb{F}$ is the concatenation of two lists: a list $\mathcal{L}_{\text {fin }}$ of positive integer powers of monic irreducible polynomials of degree at least 1 with coefficients in $\mathbb{F}$ and a list $\mathcal{L}_{\infty}$ of elementary divisors $\mu^{\alpha_{1}}, \mu^{\alpha_{2}}, \ldots, \mu^{\alpha_{g \infty}}$ at $\infty$. The length of the longest sublist of $\mathcal{L}_{\text {fin }}$ containing powers of the same irreducible polynomial is denoted by $g_{\text {fin }}(\mathcal{L})$ and the length of $\mathcal{L}_{\infty}$ is denoted by $g_{\infty}(\mathcal{L})$. The sum of the degrees of the elements in $\mathcal{L}_{\text {fin }}$ is denoted by $\delta_{\text {fin }}(\mathcal{L})$ and the sum of the degrees of the elements in $\mathcal{L}_{\infty}$ is denoted by $\delta_{\infty}(\mathcal{L})$.

TheOrem 3.12. Let $\mathcal{L}$ be a list of elementary divisors over an infinite field $\mathbb{F}$, let $\mathcal{M}_{r}=\left\{\varepsilon_{1}, \ldots, \varepsilon_{p}\right\}$ be a list of right minimal indices, let $\mathcal{M}_{l}=\left\{\eta_{1}, \ldots, \eta_{q}\right\}$ be a list of left minimal indices, and define

$$
S(\mathcal{L})=\delta_{\text {fin }}(\mathcal{L})+\delta_{\infty}(\mathcal{L})+\sum_{i=1}^{q} \eta_{i}+\sum_{i=1}^{p} \varepsilon_{i} .
$$

Then, there exists a matrix polynomial $P(\lambda)$ with coefficients in $\mathbb{F}$ of degree $d$ and whose elementary divisors, right minimal indices, and left minimal indices are, respectively, those in the lists $\mathcal{L}, \mathcal{M}_{r}$, and $\mathcal{M}_{l}$ if and only if 

(i) $d$ is a divisor of $S(\mathcal{L})$,
(ii) $\frac{S(\mathcal{L})}{d} \geq g_{\text {fin }}(\mathcal{L})$, and
(iii) $\frac{S(\mathcal{L})}{d}>g_{\infty}(\mathcal{L})$.

In this case, the rank of $P(\lambda)$ is $S(\mathcal{L}) / d$ and the size of $P(\lambda)$ is $(q+S(\mathcal{L}) / d) \times(p+$ $S(\mathcal{L}) / d)$.

Proof. ( $\Longrightarrow$ ) Let us assume that $P(\lambda)$ exists. Then Theorem 3.1 implies $d \operatorname{rank}(P)$ $=S(\mathcal{L})$, so, $(3.26)(\mathrm{i})$ follows and $\operatorname{rank}(P)=S(\mathcal{L}) / d$. The nontrivial invariant polynomials of $P(\lambda)$ are uniquely determined from the elementary divisors in the sublist $\mathcal{L}_{\text {fin }}$ of $\mathcal{L}$, as explained, for instance, in [13, Chapter VI, p. 142], and its number is precisely $g_{\text {fin }}(\mathcal{L})$. Now (3.26)(ii) follows from $\operatorname{rank}(P) \geq g_{\text {fin }}(\mathcal{L})$. Finally, (3.26)(iii) follows from Lemma 2.7. The size of $P(\lambda)$ is obtained from the rank-nullity theorem.

$(\Longleftarrow)$ Let us assume that the three conditions in $(3.26)$ hold and note that $(3.26)($ iii) guarantees that $S(\mathcal{L})>0$. From $(3.26)(\mathrm{i})$ define the positive integer $r:=S(\mathcal{L}) / d$.

First, let us denote for simplicity $g_{\mathrm{fin}}:=g_{\mathrm{fin}}(\mathcal{L})$ and construct with all the elements in $\mathcal{L}_{\text {fin }}$ a sequence of monic polynomials $q_{j}(\lambda), j=1, \ldots, g_{\text {fin }}$, of degree larger than zero and such that $q_{j}(\lambda)$ is a divisor of $q_{j+1}(\lambda)$ for $j=1, \ldots, g_{\mathrm{fin}}-1$. This construction is easy and is explained in [13, Chapter VI, p. 142]. Taking into account that $r-g_{\mathrm{fin}} \geq 0$ by (3.26)(ii), we define the following list of $r$ polynomials:

$$
\left(p_{1}(\lambda), \ldots, p_{r}(\lambda)\right)=(\underbrace{1, \ldots, 1}_{r-g_{\mathrm{fin}}}, q_{1}(\lambda), \ldots, q_{g_{\mathrm{fin}}}(\lambda)) .
$$

Observe that the sum of the degrees of $p_{1}(\lambda), \ldots, p_{r}(\lambda)$ is precisely $\delta_{\text {fin }}(\mathcal{L})$.

Second, denote for simplicity $g_{\infty}:=g_{\infty}(\mathcal{L})$ and let $\alpha_{1} \leq \alpha_{2} \leq \cdots \leq \alpha_{g_{\infty}}$ be the exponents of the elements of $\mathcal{L}_{\infty}$, which are all positive. Taking into account that $r-g_{\infty}>0$ by (3.26)(iii), we define the following list of $r$ nonnegative numbers:

$$
\left(\gamma_{1}, \ldots, \gamma_{r}\right)=(\underbrace{0, \ldots, 0}_{r-g_{\infty}}, \alpha_{1}, \alpha_{2}, \ldots, \alpha_{g_{\infty}}) .
$$

Observe that $\sum_{i=1}^{r} \gamma_{i}=\delta_{\infty}(\mathcal{L})$ and that $\gamma_{1}=0$.

Finally, note that if we take $r=S(\mathcal{L}) / d$, as defined before, $m:=q+S(\mathcal{L}) / d$, and $n:=p+S(\mathcal{L}) / d$, then $(3.25)$ becomes precisely (3.1) for the polynomials defined in (3.27) and the partial multiplicity sequence at $\infty$ given in (3.28), and therefore the existence of $P(\lambda)$ follows from Theorem 3.3.

4. Existence and possible sizes and eigenstructures of $\ell$-ifications. The new concept of $\ell$-ification of a matrix polynomial has been recently introduced in [10, section 3] as a natural generalization of the classical definition of linearization [14]. One motivation for investigating $\ell$-ifications is that there exist matrix polynomials with special structures arising in applications which do not have any linearization with the same structure [10, section 7]. The results introduced in section 3 allow us to establish easily in this section a number of new results on $\ell$-ifications that would be difficult to prove by other means. Let us start by recalling the definition of $\ell$-ification.

Definition 4.1. A matrix polynomial $R(\lambda)$ of degree $\ell>0$ is said to be an $\ell$-ification of a given matrix polynomial $P(\lambda)$ if for some $r, s \geq 0$ there exist unimodular matrix polynomials $U(\lambda)$ and $V(\lambda)$ such that

$$
U(\lambda)\left[\begin{array}{ll}
R(\lambda) & \\
& I_{s}
\end{array}\right] V(\lambda)=\left[\begin{array}{ll}
P(\lambda) & \\
& I_{r}
\end{array}\right] .
$$


If, in addition, rev $R(\lambda)$ is an $\ell$-ification of rev $P(\lambda)$, then $R(\lambda)$ is said to be a strong $\ell$-ification of $P(\lambda)$.

In [10], $\ell$-ifications are defined to have degree smaller than or equal to $\ell$. However, since in most cases it is of interest to fix the exact degree of a matrix polynomial, we focus in this paper on $\ell$-ifications with degree $\ell$. It is natural to think that in applications the most interesting $\ell$-ifications of $P(\lambda)$ should satisfy $\ell<\operatorname{deg}(P)$, but observe that the results presented in this section do not require such an assumption and so they will be stated for any positive integer $\ell$. This is one of the reasons for considering two identity matrices $I_{s}$ and $I_{r}$ in Definition 4.1, although only one of them is really needed [10, Corollary 4.4]. Observe also that, since we assume $\ell>0$, zero degree polynomials are never considered $\ell$-ifications in this work.

In this section we consider two matrix polynomials, $P(\lambda)$ and $R(\lambda)$, so it is convenient to adopt for brevity the following notation: for any matrix polynomial $Q(\lambda)$, $\delta_{\text {fin }}(Q)$ is the sum of the degrees of the invariant polynomials of $Q(\lambda), \delta_{\infty}(Q)$ is the sum of the degrees of the elementary divisors at $\infty$ of $Q(\lambda), g_{\mathrm{fin}}(Q)$ is the number of nontrivial invariant polynomials of $Q(\lambda)$ (equivalently, the largest of the geometric multiplicities of the finite eigenvalues of $Q(\lambda)$ ), and $g_{\infty}(Q)$ is the number of elementary divisors of $Q(\lambda)$ at $\infty$ (equivalently, the geometric multiplicity of the infinite eigenvalue of $Q(\lambda)$ ). We will often use this notation without explicitly referring to it.

The results of this section generalize to $\ell$-ifications of arbitrary degree what was proved in Theorems 4.10 and 4.12 of [10] only for linearizations. In addition, we solve for arbitrary infinite fields the conjecture posed in [10, Remark 7.6]. In the proofs of this section, we will often use Theorem 4.1 in [10]. Therefore, we state below this result in a form specially adapted to our purposes.

Theorem 4.2 (characterization of $\ell$-ifications [10, Theorem 4.1]). Consider a matrix polynomial $P(\lambda)$ and another matrix polynomial $R(\lambda)$ of degree $\ell>0$, and the following three conditions on $P(\lambda)$ and $R(\lambda)$ :

(a) $\operatorname{dim} \mathcal{N}_{r}(P)=\operatorname{dim} \mathcal{N}_{r}(R)$ and $\operatorname{dim} \mathcal{N}_{\ell}(P)=\operatorname{dim} \mathcal{N}_{\ell}(R)$ (i.e., $P(\lambda)$ and $R(\lambda)$ have the same numbers of right and left minimal indices).

(b) $P(\lambda)$ and $R(\lambda)$ have exactly the same finite elementary divisors.

(c) $P(\lambda)$ and $R(\lambda)$ have exactly the same infinite elementary divisors. Then,

(1) $R(\lambda)$ is an $\ell$-ification of $P(\lambda)$ if and only if conditions (a) and (b) hold;

(2) $R(\lambda)$ is a strong $\ell$-ification of $P(\lambda)$ if and only if conditions (a), (b), and (c) hold.

Observe that condition (b) in Theorem 4.2 is equivalent to " $P(\lambda)$ and $R(\lambda)$ have exactly the same nontrivial invariant polynomials."

4.1. Results for standard $\ell$-ifications. Theorem 4.3 determines all possible sizes, all possible nonzero partial multiplicities at $\infty$, and all possible minimal indices of (possibly not strong) $\ell$-ifications of a given matrix polynomial $P(\lambda)$. Recall that according to Theorem 4.2 the nontrivial invariant polynomials as well as the numbers of left and right minimal indices of any $\ell$-ification of $P(\lambda)$ are the same as those of $P(\lambda)$. In Theorems 4.3 and 4.10 we use the standard Gauss bracket $\lceil x\rceil$ to denote the smallest integer larger than or equal to the real number $x$.

TheOrem 4.3 (size range of $\ell$-ifications). Let $P(\lambda)$ be an $m \times n$ matrix polynomial over an infinite field $\mathbb{F}$ with rank equal to $r>0$, let $\ell$ be a positive integer, and let

$$
\widetilde{r}=\max \left\{\left\lceil\frac{\delta_{\text {fin }}(P)}{\ell}\right\rceil, g_{\text {fin }}(P)\right\} .
$$

Copyright (? by SIAM. Unauthorized reproduction of this article is prohibited. 
Then the following hold:

(a) There is an $s_{1} \times s_{2}$ l-ification of $P(\lambda)$ if and only if

$$
s_{1} \geq(m-r)+\widetilde{r}, \quad s_{2} \geq(n-r)+\widetilde{r}, \quad \text { and } \quad s_{1}-s_{2}=m-n .
$$

In particular, the minimum-size $\ell$-ification of $P(\lambda)$ has sizes $s_{1}=(m-r)+\widetilde{r}$ and $s_{2}=(n-r)+\widetilde{r}$.

(b) Let $0 \leq \widetilde{\eta}_{1} \leq \cdots \leq \widetilde{\eta}_{m-r}$ be a list of left minimal indices, let $0 \leq \widetilde{\varepsilon}_{1} \leq \cdots \leq$ $\widetilde{\varepsilon}_{n-r}$ be a list of right minimal indices, let $0<\widetilde{\gamma}_{1} \leq \cdots \leq \widetilde{\gamma}_{t}$ be a (possibly empty) list of nonzero partial multiplicities at $\infty$, and define

$$
\widetilde{S}=\delta_{\text {fin }}(P)+\sum_{i=1}^{t} \widetilde{\gamma}_{i}+\sum_{i=1}^{m-r} \widetilde{\eta}_{i}+\sum_{i=1}^{n-r} \widetilde{\varepsilon}_{i} .
$$

Then, there exists an $\ell$-ification $R(\lambda)$ of $P(\lambda)$ having the previous lists of minimal indices and nonzero partial multiplicities at $\infty$ if and only if

$$
\text { (i) } \ell \text { is a divisor of } \widetilde{S} \text {, (ii) } \frac{\widetilde{S}}{\ell} \geq g_{\text {fin }}(P) \text {, and (iii) } \frac{\widetilde{S}}{\ell}>t \text {. }
$$

The size of this $R(\lambda)$ is $s_{1} \times s_{2}$, where

$$
s_{1}=(m-r)+\frac{\widetilde{S}}{\ell} \quad \text { and } \quad s_{2}=(n-r)+\frac{\widetilde{S}}{\ell} .
$$

Proof. (a) $(\Rightarrow)$ If $R(\lambda)$ has size $s_{1} \times s_{2}$ and is an $\ell$-ification of $P(\lambda)$, then $R(\lambda)$ has the same nontrivial invariant polynomials as $P(\lambda)$ and the same numbers, $m-r$ and $n-r$, of left and right minimal indices as $P(\lambda)$ by Theorem 4.2(1). Therefore, Theorem 3.1 applied to $R(\lambda)$ implies $\operatorname{rank}(R) \geq \delta_{\text {fin }}(R) / \ell=\delta_{\text {fin }}(P) / \ell$, and Definition 2.3 implies $\operatorname{rank}(R) \geq g_{\mathrm{fin}}(R)=g_{\mathrm{fin}}(P)$. Therefore, $\operatorname{rank}(R) \geq \widetilde{r}$. Finally, from the rank-nullity theorem, we get

$s_{1}=(m-r)+\operatorname{rank}(R) \geq(m-r)+\widetilde{r} \quad$ and $\quad s_{2}=(n-r)+\operatorname{rank}(R) \geq(n-r)+\widetilde{r}$, and $s_{1}-s_{2}=m-n$.

(a) $(\Leftarrow)$ To see that there exists an $\ell$-ification of $P(\lambda)$ for each size $s_{1} \times s_{2}$ allowed by (4.1), note first that each of these sizes can be written as

$$
s_{1}=(m-r)+\widehat{r}, \quad s_{2}=(n-r)+\widehat{r},
$$

for some $\widehat{r} \geq \widetilde{r}$. Therefore, $\widehat{r} \geq \delta_{\text {fin }}(P) / \ell$, and so there are nonnegative numbers $0 \leq \widetilde{\eta}_{1} \leq \cdots \leq \widetilde{\eta}_{m-r}$ and $0 \leq \widetilde{\varepsilon}_{1} \leq \cdots \leq \widetilde{\varepsilon}_{n-r}$ such that

$$
\ell \widehat{r}=\delta_{\text {fin }}(P)+\sum_{i=1}^{m-r} \widetilde{\eta}_{i}+\sum_{i=1}^{n-r} \widetilde{\varepsilon}_{i} .
$$

In addition, $\widehat{r} \geq g_{\mathrm{fin}}(P)$. Combining all this information with Theorem 3.12, we get that there exists a matrix polynomial $R(\lambda)$ of degree $\ell$, with no elementary divisors at $\infty$, with the same nontrivial invariant polynomials ${ }^{3}$ as $P(\lambda)$, with left and right

\footnotetext{
${ }^{3}$ Recall that there is a bijection between the set of lists of finite elementary divisors and the set of lists of nontrivial invariant polynomials [13, Chapter VI, p. 142].
}

Copyright $\odot$ by SIAM. Unauthorized reproduction of this article is prohibited. 
minimal indices equal, respectively, to $0 \leq \widetilde{\eta}_{1} \leq \cdots \leq \widetilde{\eta}_{m-r}$ and $0 \leq \widetilde{\varepsilon}_{1} \leq \cdots \leq \widetilde{\varepsilon}_{n-r}$, and with size $s_{1} \times s_{2}$ such that

$$
s_{1}=(m-r)+\widehat{r} \quad \text { and } \quad s_{2}=(n-r)+\widehat{r} .
$$

This $R(\lambda)$ is an $\ell$-ification of $P(\lambda)$ by Theorem $4.2(1)$.

(b) It is an immediate consequence of Theorem 3.12 and Theorem 4.2(1).

Remark 4.4. Observe that Theorem 4.3(a) guarantees, in particular, that every matrix polynomial has an $\ell$-ification for any positive degree $\ell$. Note, in addition, that $\ell$-ifications can have arbitrarily large sizes. The three conditions in (4.1) are redundant, since the second one follows from the first and the third ones. We write all of them to make explicit both minimum sizes of $\ell$-ifications.

Remark 4.5. In Theorem 4.3(b) the cardinalities of the lists of left and right minimal indices are $m-r$ and $n-r$, respectively, and so they are determined by the size and the rank of $P(\lambda)$. However, the cardinality $t$ of the list of nonzero partial multiplicities at $\infty$ can be chosen, and, in particular, this list can be chosen to be empty.

Remark 4.6. Let $0 \leq \eta_{1} \leq \cdots \leq \eta_{m-r}$ be the left minimal indices of $P(\lambda)$, let $0 \leq$ $\varepsilon_{1} \leq \cdots \leq \varepsilon_{n-r}$ be the right minimal indices of $P(\lambda)$, and let $0<\gamma_{1} \leq \cdots \leq \gamma_{g_{\infty}(P)}$ be the nonzero partial multiplicities at $\infty$ of $P(\lambda)$, and define

$$
S=\delta_{\text {fin }}(P)+\sum_{i=1}^{g_{\infty}(P)} \gamma_{i}+\sum_{i=1}^{m-r} \eta_{i}+\sum_{i=1}^{n-r} \varepsilon_{i} .
$$

Theorem 4.3(b) implies that if $\ell$ is a divisor of $S$, and if $S / \ell \geq g_{\mathrm{fin}}(P)$ and $S / \ell>$ $g_{\infty}(P)$, then there exists an $\ell$-ification $R(\lambda)$ of $P(\lambda)$ with exactly the same complete eigenstructure as $P(\lambda)$ (barring trivial invariant polynomials). In particular, this always happens if $\ell$ is a divisor of $d=\operatorname{deg}(P(\lambda))$, since in this case Theorem 3.1 implies $d r=S$ and so $S / \ell \geq r$, which can be combined with $r \geq g_{\text {fin }}(P)$ and $r>g_{\infty}(P)$ (see Lemma 2.7) to show that the three conditions in (4.2) hold. Observe that such $R(\lambda)$ is in fact a strong $\ell$-ification of $P(\lambda)$ according to Theorem 4.2(2).

In particular, Theorem 4.3(b) implies that there always exist linearizations (just make $\ell=1$ in the discussion above) which preserve the complete eigenstructure of $P(\lambda)$. This result was already obtained in [35], although expressed in a different language. An advantage of the approach in [35] is that a construction of such linearizations was provided there. It is an interesting open problem to derive such a construction for the case of $\ell$-ifications (with $\ell>1$ ) that preserve the complete eigenstructure of $P(\lambda)$ under the conditions mentioned in the previous paragraph.

4.2. Results for strong $\ell$-ifications of regular polynomials. Our next result generalizes Theorem 7.5 in [10] in two senses. First, Theorem 4.7 is valid for strong $\ell$-ifications of any degree, i.e., smaller than, equal to, or larger than the degree of $P(\lambda)$, while Theorem 7.5 in [10] is valid only for strong $\ell$-ifications with degree smaller than or equal to the degree of $P(\lambda)$. Second, Theorem 4.7 is valid for arbitrary infinite fields, while Theorem 7.5 in [10] is valid only for algebraically closed fields.

THEOREM 4.7 (size of strong $\ell$-fications of regular polynomials). Let $P(\lambda)$ be an $n \times n$ regular matrix polynomial over an infinite field $\mathbb{F}$ of degree $d$ and let $\ell$ be a positive integer. Then, there exists a strong $\ell$-ification $R(\lambda)$ of $P(\lambda)$ if and only if

(i) $\ell$ is a divisor of $d n$, (ii) $\frac{d n}{\ell} \geq g_{\text {fin }}(P)$, and (iii) $\frac{d n}{\ell}>g_{\infty}(P)$. 
In addition, the size of any strong $\ell$-ification of $P(\lambda)$ is $(d n) / \ell \times(d n) / \ell$. In particular, if $\ell>d n$, then there are no strong $\ell$-ifications of $P(\lambda)$.

Proof. Recall throughout the proof that Theorem 3.1 implies that $d n=\delta_{\text {fin }}(P)+$ $\delta_{\infty}(P)$, since $P(\lambda)$ is regular and has no minimal indices.

First, assume that the conditions in (4.3) hold. Therefore, Theorem 3.12 with $S(\mathcal{L})=\delta_{\text {fin }}(P)+\delta_{\infty}(P)$ implies that there exists a regular matrix polynomial $R(\lambda)$ of degree $\ell$ with the same nontrivial invariant polynomials as $P(\lambda)$ and with the same elementary divisors at $\infty$ as $P(\lambda)$. Such $R(\lambda)$ has size $(d n) / \ell \times(d n) / \ell$ and is a strong $\ell$-ification of $P(\lambda)$ by Theorem 4.2(2).

Next, assume that there exists a strong $\ell$-ification $R(\lambda)$ of $P(\lambda)$. According to Theorem 4.2(2), $R(\lambda)$ is regular and has the same nontrivial invariant polynomials as $P(\lambda)$ and the same elementary divisors at $\infty$ as $P(\lambda)$. Therefore, if $R(\lambda)$ has size $s \times s$, then Theorem 3.1 applied to $R(\lambda)$ implies $s \ell=\delta_{\text {fin }}(P)+\delta_{\infty}(P)$, which, together with Lemma 2.7, implies the conditions (4.3).

The following two corollaries follow easily from Theorem 4.7. Corollary 4.8 is well known: both the result on existence and the result on size (see [7, Theorem 3.2] and [10, Theorem 4.12]). We include this corollary here just to show that these classical results can be retrieved from Theorem 4.7. Corollary 4.9 shows that Theorem 4.7 simplifies considerably if we assume that $\ell \leq \operatorname{deg}(P)$. Corollary 4.9 is related to Theorem 7.5 in [10].

COROLlaRY 4.8 (size of strong linearizations of regular polynomials). For any $n \times$ $n$ regular matrix polynomial $P(\lambda)$ over an infinite field $\mathbb{F}$ of degree $d \geq 1$, there always exists a strong linearization of $P(\lambda)$ and the size of any such strong linearization is $(d n) \times(d n)$.

Proof. If $\ell=1$, then (i) in (4.3) always holds, and the same happens for (ii) and (iii), since $n \geq g_{\text {fin }}(P)$ and $n>g_{\infty}(P)$. The size follows also from Theorem 4.7.

Corollary 4.9. Let $P(\lambda)$ be an $n \times n$ regular matrix polynomial of degree $d$ over an infinite field $\mathbb{F}$ and let $1 \leq \ell \leq d$ be an integer. Then, there exists a strong $\ell$-ification of $P(\lambda)$ if and only if $\ell$ divides $d n$. In addition, the size of any strong $\ell$-ification of $P(\lambda)$ is $(d n) / \ell \times(d n) / \ell$.

Proof. The result follows from Theorem 4.7 and the following observation: $n \geq$ $g_{\mathrm{fin}}(P)$ and $n>g_{\infty}(P)$ always hold, where the last strict inequality is because of Lemma 2.7. In addition, $d / \ell \geq 1$. Therefore, (ii) and (iii) in (4.3) automatically hold in this case.

4.3. Results for strong $\ell$-ifications of singular polynomials. Theorem 4.10 determines all possible sizes and all possible minimal indices of strong $\ell$-ifications of a given singular matrix polynomial $P(\lambda)$. Recall that according to Theorem 4.2, the nontrivial invariant polynomials, the elementary divisors at $\infty$, and the numbers of left and right minimal indices of any strong $\ell$-ification of $P(\lambda)$ are the same as those of $P(\lambda)$.

THEOREM 4.10 (size range of strong $\ell$-ifications of singular polynomials). Let $P(\lambda)$ be an $m \times n$ singular matrix polynomial over an infinite field $\mathbb{F}$ with rank equal to $r>0$, i.e., at least one of $m-r$ or $n-r$ is nonzero, let $\ell$ be a positive integer, and let

$$
\widetilde{r}=\max \left\{\left\lceil\frac{\delta_{\text {fin }}(P)+\delta_{\infty}(P)}{\ell}\right\rceil, g_{\text {fin }}(P), g_{\infty}(P)+1\right\} .
$$

Then the following hold: 
(a) There is an $s_{1} \times s_{2}$ strong $\ell$-ification of $P(\lambda)$ if and only if

$$
s_{1} \geq(m-r)+\widetilde{r}, \quad s_{2} \geq(n-r)+\widetilde{r}, \quad \text { and } \quad s_{1}-s_{2}=m-n .
$$

In particular, the minimum-size strong $\ell$-ification of $P(\lambda)$ has sizes $s_{1}=$ $(m-r)+\widetilde{r}$ and $s_{2}=(n-r)+\widetilde{r}$.

(b) Let $0 \leq \widetilde{\eta}_{1} \leq \cdots \leq \widetilde{\eta}_{m-r}$ be a list of left minimal indices, let $0 \leq \widetilde{\varepsilon}_{1} \leq \cdots \leq$ $\widetilde{\varepsilon}_{n-r}$ be a list of right minimal indices, and define

$$
\widetilde{S}=\delta_{\text {fin }}(P)+\delta_{\infty}(P)+\sum_{i=1}^{m-r} \widetilde{\eta}_{i}+\sum_{i=1}^{n-r} \widetilde{\varepsilon}_{i} .
$$

Then, there exists a strong $\ell$-ification $R(\lambda)$ of $P(\lambda)$ having the previous lists of minimal indices if and only if

$$
\text { (i) } \ell \text { is a divisor of } \widetilde{S} \text {, (ii) } \frac{\widetilde{S}}{\ell} \geq g_{\text {fin }}(P) \text {, and (iii) } \frac{\widetilde{S}}{\ell}>g_{\infty}(P) \text {. }
$$

The size of this $R(\lambda)$ is $s_{1} \times s_{2}$, where

$$
s_{1}=(m-r)+\frac{\widetilde{S}}{\ell} \quad \text { and } \quad s_{2}=(n-r)+\frac{\widetilde{S}}{\ell} .
$$

Proof. The proof is essentially the same as the proof of Theorem 4.3 and is omitted. We just emphasize the key differences that have to be considered. If $R(\lambda)$ is a strong $\ell$-ification of $P(\lambda)$, then $R(\lambda)$ and $P(\lambda)$ have the same nontrivial invariant polynomials, the same numbers of left and right minimal indices, and, in addition, the same elementary divisors at $\infty$ by Theorem 4.2(2). Therefore, Theorem 3.1 applied to $R(\lambda)$ implies $\operatorname{rank}(R) \geq\left(\delta_{\text {fin }}(P)+\delta_{\infty}(P)\right) / \ell$, Definition $2.3 \operatorname{implies} \operatorname{rank}(R) \geq g_{\text {fin }}(P)$, and Lemma 2.7 implies $\operatorname{rank}(R)>g_{\infty}(P)$. Then, the role of $\delta_{\text {fin }}(P)$ in the proof of Theorem 4.3 is played here by $\delta_{\text {fin }}(P)+\delta_{\infty}(P)$ and we have an additional constraint on the geometric multiplicity at $\infty$.

Remark 4.11. Observe that Theorem 4.10(a) guarantees, in particular, that every singular matrix polynomial has a strong $\ell$-fication for any degree $\ell$. Note, in addition, that strong $\ell$-ifications of singular polynomials can have arbitrarily large sizes. These two results are in stark contrast to the situation for regular matrix polynomials, since Theorem 4.7 shows that, in this case, strong $\ell$-ifications exist only for certain values of $\ell$ and that their sizes are fixed.

5. Conclusions. We have solved a very general inverse problem for matrix polynomials with completely prescribed eigenstructure. As far as we know, this is the first result of this type that considers the complete eigenstructure, i.e., both regular and singular, of a matrix polynomial of arbitrary degree. The solution of this inverse problem has been used to settle the existence problem of $\ell$-fications of a given matrix polynomial as well as to determine their possible sizes and eigenstructures.

The results presented in this work lead naturally to many related open problems, for instance, the solution of inverse problems for structured matrix polynomials with completely prescribed eigenstructure for classes of polynomials that are important in applications, such as symmetric, skew-symmetric, alternating, and palindromic matrix polynomials. Another open problem in this area is to develop efficient methods for constructing, when possible, matrix polynomials of a given degree, with given lists of finite and infinite elementary divisors, and with given lists of left and right minimal 
indices, since the construction used in the proof of Theorem 3.3 is not efficient in practice. This is closely related to the problem of constructing strong $\ell$-ifications in the situations that are not covered by the $\ell$-ifications presented in [10, section 5]. Finally, the problem of extending to finite fields the results of this paper is still open as well.

Acknowledgments. The authors thank Steve Mackey for many illuminating discussions, held over many years, on matrix polynomial inverse (or "realizability") eigenvalue problems. In particular, Theorem 3.12 is a general version, valid for arbitrary degrees, of the result that Steve has proved for degree two via completely different techniques in [20]. The authors also thank two anonymous referees for many constructive suggestions that have contributed to improving the paper considerably. The scientific responsibility of this work rests with its authors.

\section{REFERENCES}

[1] A. Amparan, S. Marcaida, And I. Zaballa, Local realizations and local polynomial matrix representations of systems, Linear Algebra Appl., 425, (2007) pp. 757-775.

[2] E. N. Antoniou And S. Vologiannidis, A new family of companion forms of polynomial matrices, Electron. J. Linear Algebra, 11 (2004), pp. 78-87.

[3] Z. BAI AND Y. Su, SOAR: A second-order Arnoldi method for the solution of the quadratic eigenvalue problem, SIAM J. Matrix Anal. Appl., 26 (2005), pp. 640-659.

[4] T. Betcke and D. Kressner, Perturbation, extraction and refinement of invariant pairs for matrix polynomials, Linear Algebra Appl., 435 (2011) pp. 574-536.

[5] D. Bini And V. Noferini, Solving polynomial eigenvalue problems by means of the EhrlichAberth method, Linear Algebra Appl., 439 (2013) pp. 1130-1149.

[6] Y. CAI AND S. Xu, On a quadratic inverse eigenvalue problem, Inverse Problems, 25 (2009), 085004.

[7] F. De Terán and F. M. Dopico, Sharp lower bounds for the dimension of linearizations of matrix polynomials, Electron. J. Linear Algebra, 17 (2008), pp. 518-531.

[8] F. De Terán, F. M. Dopico, and D. S. Mackey, Linearizations of singular matrix polynomials and the recovery of minimal indices, Electron. J. Linear Algebra, 18 (2009), pp. 371-402.

[9] F. De Terán, F. M. Dopico, and D. S. Mackey, Fiedler companion linearizations and the recovery of minimal indices, SIAM J. Matrix Anal. Appl., 31 (2010), pp. 2181-2204.

[10] F. De Terán, F. M. Dopico, And D. S. Mackey, Spectral equivalence of matrix polynomials and the index sum theorem, Linear Algebra Appl., 459 (2014), pp. 264-333.

[11] F. De Terán, F. M. Dopico, D. S. Mackey, and V. Perović, Quadratic realizability of palindromic matrix polynomials, in preparation.

[12] G. D. Forney, Minimal bases of rational vector spaces, with applications to multivariable linear systems, SIAM J. Control, 13 (1975), pp. 493-520.

[13] F. Gantmacher, The Theory of Matrices, Chelsea, New York, 1959.

[14] I. Gohberg, P. Lancaster, and L. Rodman, Matrix Polynomials, Classics in Appl. Math., SIAM, Philadelphia, 2009.

[15] S. Hammarling, C. Munro, and F. Tisseur, An algorithm for the complete solution of quadratic eigenvalue problems, ACM Trans. Math. Software, 39 (2013), pp. 18:1-18:19.

[16] R. A. Horn and C. R. Johnson, Matrix Analysis, Cambridge University Press, Cambridge, UK, 1985.

[17] S. Johansson, B. KÅgström, and P. Van Dooren, Stratification of full rank polynomial matrices, Linear Algebra Appl., 439 (2013), pp. 1062-1090

[18] T. Kailath, Linear Systems, Prentice-Hall, Englewood Cliffs, NJ, 1980.

[19] P. LANCASTER AND I. Zaballa, On the inverse symmetric quadratic eigenvalue problem, SIAM J. Matrix Anal. Appl., 35 (2014), pp. 254-278.

[20] D. S. MACKEY, A quasi-canonical form for quadratic matrix polynomials, in preparation.

[21] D. S. Mackey, N. Mackey, C. Mehl, and V. Mehrmann, Vector spaces of linearizations for matrix polynomials, SIAM J. Matrix Anal. Appl., 28 (2006), pp. 971-1004.

[22] D. S. Mackey, N. Mackey, C. Mehl, and V. Mehrmann, Möbius transformations of matrix polynomials, Linear Algebra Appl., 470 (2015), pp. 120-184.

[23] E. Marques de SÁ, Imbedding conditions for $\lambda$-matrices, Linear Algebra Appl., 24 (1979), pp. $33-50$. 
[24] K. Meerbergen, The quadratic Arnoldi method for the solution of the quadratic eigenvalue problem, SIAM J. Matrix Anal. Appl., 30 (2008), pp. 1463-1482.

[25] W. Neven and C. Praagman, Column reduction of polynomial matrices, Linear Algebra Appl., 188-189 (1993), pp. 569-589.

[26] M. Newman, Integral Matrices, Academic Press, New York, 1972.

[27] V. NofERINI, The behavior of the complete eigenstructure of a polynomial matrix under a generic rational transformation, Electron. J. Linear Algebra, 23 (2012), pp. 607-624.

[28] C. PraAgman, Invariants of polynomial matrices, in Proceedings of ECC91, Grenoble, 1991, pp. 1274-1277.

[29] H. H. RosenBrock, State-space and Multivariable Theory, Thomas Nelson and Sons, London, 1970.

[30] L. Taslaman, F. Tisseur, and I. Zaballa, Triangularizing matrix polynomials, Linear Algebra Appl., 439 (2013), pp. 1679-1699.

[31] R. C. Thompson, Smith invariants of a product of integral matrices, Contemp. Math. AMS, 47 (1985), pp. 401-434.

[32] F. Tisseur And K. Meerbergen, The quadratic eigenvalue problem, SIAM Rev., 43 (2001), pp. $235-286$.

[33] F. Tisseur and I. Zaballa, Triangularizing Quadratic Matrix Polynomials, SIAM. J. Matrix Anal. Appl., 34 (2013), pp. 312-337.

[34] P. Van Dooren, Reducing subspaces: Definitions, properties, and algorithms, in Matrix Pencils, Lecture Notes in Math. 973, B. Kågström and A. Ruhe, eds., Springer-Verlag, Berlin, 1983, pp. 58-73.

[35] P. VAn Dooren ANd P. Dewilde, The eigenstructure of an arbitrary polynomial matrix: Computational aspects, Linear Algebra Appl., 50 (1983), pp. 545-579.

[36] G. Verghese, P. Van Dooren, and T. Kailath, Properties of the system matrix of a generalized state-space system, Internat. J. Control, 30 (1979), pp. 235-243.

[37] J. H. Wilkinson, Linear differential equations and Kronecker's canonical form, in Recent Advances in Numerical Analysis. C. de Boor and G. Golub, eds., Academic Press, New York, 1978, pp. 231-265.

Copyright (? by SIAM. Unauthorized reproduction of this article is prohibited. 